FERMILAB-TM-1900

\title{
High Brightness Symmetric Emittance RF Photoinjector Preliminary Design Report
}

\author{
Eric R. Colby and Jean-François Ostiguy \\ Fermi National Accelerator Laboratory \\ P.O. Box 500, Batavia, Illinois 60510 \\ James B. Rosenzweig \\ University of California, Los Angeles \\ 405 Hilgard Avenue \\ Los Angeles, CA 90024
}

August 1994 


\section{Disclaimer}

This report was prepared as an account of work sponsored by an agency of the United States Government. Neither the United States Government nor any agency thereof, nor any of their employees, makes any warranty, express or implied, or assumes any legal liability or responsibility for the accuracy, completeness, or usefulness of any information, apparatus, product, or process disclosed, or represents that its use would not infringe privately owned rights. Reference herein to any specific commercial product, process, or service by trade name, trademark, manufacturer, or otherwise, does not necessarily constitute or imply its endorsement, recommendation, or favoring by the United States Government or any agency thereof. The views and opinions of authors expressed herein do not necessarily state or reflect those of the United States Government or any agency there of. 


\title{
High Brightness Symmetric Emittance RF Photoinjector Preliminary Design Report
}

\author{
Eric R. Colby \\ Fermi National Accelerator Laboratory/University of California, Los Angeles \\ Mail Station 345 \\ P. O. Box 500 \\ Batavia, Illinois 60510-0500 \\ Jean-François Ostiguy \\ Fermi National Accelerator Laboratory \\ Mail Station 345 \\ P. O. Box 500 \\ Batavia, Illinois $60510-0500$ \\ James B. Rosenzweig \\ University of California, Los Angeles \\ Department of Physics \\ 405 Hilgard Avenue \\ Los Angeles, CA 90024
}

September 15, 1994

\begin{abstract}
A preliminary design for a high bunch charge $(8 \mathrm{nC})$, low emittance $(<20 \mathrm{~mm}-\mathrm{mr})$ radiofrequency electron photoinjector matched to the requirements of the Tesla Test Facility is presented. A 1.5 cell iris coupled $\pi$-mode structure with high average accelerating gradient is chosen for its high shunt impedance, simplicity, and ability to accommodate an externally mounted solenoid for simultaneous beam divergence control and emittance compensation. Beam optics are optimized for an overall injector consisting of the electron gun followed by one linac capture section, a dipole chicane for magnetic bunch compression to achieve a bunch length corresponding to $\sigma_{z}=1 \mathrm{~mm}$. Electrical and beam dynamical aspects of the photoinjector design are presented. A description of the proposed experimental program is included.
\end{abstract}




\section{Introduction}

The needs for excellent beam quality and high peak current beams for free electron laser (FEL) applications have led to the development of the radiofrequency (RF) electron photoinjector as a high brightness electron source. The demands of a high luminosity linear collider similarly require excellent beam quality, although usually achieved with the aid of a damping ring. The development of normal and superconducting linac structures for linear colliders requires testing the structures under conditions as similar to the planned operating conditions as possible. Wakefield analysis, beam loading, multi-bunch instabilities and other beam-cavity interactions require beams of similar charge, bunch length, bunch spacing, and approximate transverse emittance as the eventual collider beam. Analysis of the superconducting structures for the Tera-Electron volt Superconducting Linear Accelerator (TESLA), normal conducting structures for the Next Linear Collider (NLC), or for a proposed [3] superconducting injector for the Fermilab Main Injector will all require high brightness test beams. The construction of an injector to achieve the needed beam quality will require state-of-the-art accelerator technology. To this end, we discuss the applicability of an RF electron photoinjector as a very compact source of high quality beams with high bunch charge, very short bunch length, that is capable of high repetition rates (better than $1 \mathrm{MHz}$ ).

The design for a photoinjector satisfying the demands of the TESLA Test Facility (TTF) is presented below. Current TTF and TESLA500 (the next design phase of the TESLA program) requirements are summarized in table 1 below.

\begin{tabular}{|l|c|l|}
\hline Parameter & Symbol & Value \\
\hline \hline Bunches per macropulse & $N_{m}$ & $1000(800)$ \\
\hline Macropulse spacing & $\tau_{m}$ & $100 \mathrm{~ms}$ \\
\hline Bunch spacing & $\tau_{b}$ & $1 \mu s$ \\
\hline Overall Duty Cycle & & $1 \%$ \\
\hline Bunch Charge & $Q_{b}$ & $8.0 \mathrm{nC}$ \\
\hline Bunch length & $\sigma_{b}$ & $1 \mathrm{~mm}$ \\
\hline Peak Current & $I_{p}$ & $958 \mathrm{~A}$ \\
\hline RF Frequency & $f_{R F}$ & $1.3 \mathrm{GHz}$ \\
\hline RF Power per klystron & $P_{R F}$ & $4.5 \mathrm{MW}$ \\
\hline TESLA Test Facility & & Symmetric \\
\hline Horizontal Emittance & $\epsilon_{x}$ & $<20 \pi \mathrm{mm}-\mathrm{mr}$ \\
\hline Vertical Emittance & $\epsilon_{y}$ & $<20 \pi \mathrm{mm}-\mathrm{mr}$ \\
\hline Brightness & $B$ & $4.8 \times 10^{12} \mathrm{~A} / \mathrm{m}^{2}$ \\
\hline TESLA500 & & $\mathbf{A s y m m e t r i c}$ \\
\hline Horizontal Emittance & $\epsilon_{x}$ & $20 \pi \mathrm{mm}-\mathrm{mr}$ \\
\hline Vertical Emittance & $\epsilon_{y}$ & $1 \pi \mathrm{mm}-\mathrm{mr}$ \\
\hline Brightness & $B$ & $95.8 \times 10^{12} \mathrm{~A} / \mathrm{m}^{2}$ \\
\hline
\end{tabular}

Table 1: Summary of TESLA Injector II Parameters 
The experimental program to construct and commission the photoinjector will have three phases. The first phase involves the testing of the photoinjector under essentially single-pulse operation to address single bunch beam dynamics issues, and to complete the optimization of the emittance compensation and magnetic compression assemblies. The beamline outlined below is chosen with flexibility in mind to permit some exploration of parameters, including high-gradient operation of the photoinjector, obviating the need for a magnetic compression chicane altogether, and different emittance compensation lens strengths and positions. Optics for splitting and delaying the photocathode excitation laser pulse to provide a train of as many as ten pulses will be fitted towards the end of phase I to begin the study of wakefield and beam loading effects. Phase I forms part of an established research and development collaboration between Fermilab and UCLA to address beam dynamics issues of very high brightness symmetric and asymmetric emittance electron photoinjectors. Beam physics results are expected for both symmetric and asymmetric injectors by mid-1995.

Phase II will commence with the installation of the prototype laser system, which is presently under study by both industry and colleagues at the Max Born Institut für Physik in Berlin. Industry has already demonstrated both the technology and the willingness to pursue the design of the laser system. The three stage Ti:Sa/LiSaF system will produce the required train of 1000 pulses spaced $1 \mu$ s apart, allowing testing of the photoinjector under conditions identical to those of the TESLA500 linear collider. Appendix B details the requirements of the photocathode drive laser. Funding and the precise scheduling of activities during phase II have yet to be fully decided.

Phase III entails delivery of the laser system, photoinjector, linac section, magnetic compression chicane and diagnostics to the TTF at DESY in the winter of 1996-97. Commissioning of the photoinjector assembly at DESY will complete the symmetric emittance photoinjector project.

\section{Beam Dynamics Considerations}

The need for substantial bunch charge with good beam quality requires that the RF accelerating gradient in the photoinjector be relatively high to reduce space charge induced emittance growth, and that emittance compensation described by Carlsten et al [4] be implemented to reduce the correlated space charge emittance growth. RF contributions to the emittance must be carefully controlled, requiring that nonlinear variations of the accelerating field in both the radial and longitudinal direction be minimized, and that the beam dimensions and accelerating gradient be carefully optimized.

The Carlsten emittance compensation scheme requires that the bunch be given a focussing kick early on to start the gradual reclosure of the phase space "fan" that results from the longitudinal variation of the transverse space charge fields. The variation of the space charge fields results in a correlated emittance growth that can be partially reversed with a focussing kick from a linear lens. Ideally, the kick should take place ahead of the beam's exit from the RF photoinjector, as the time-dependent defocussing that takes place within the RF structure, which can interfere with the proper implementation of emittance compensation, depends on the square of the transverse beam size [9] and will be significantly smaller if the beam is focussed as close to the cathode as possible. As the emittance 
compensation scheme is sensitive both to the strength and to the longitudinal position of the focussing kick, a second solenoid, downstream of the RF feed for the full cell, is added to allow exploration of the efficacy of the compensation as a function of lens strength and position, with the ratio of the currents in the upstream and downstream solenoids determining the effective magnetic center of the lens, and the sum of the currents determining the overall focal length. A bucking coil to cancel the longitudinal magnetic field on the cathode completes the focussing assembly. Figure 1 provides a schematic representation of where the three solenoids are positioned. The bevelled edge on the downstream side of the first focussing solenoid is to accomodate two laser ports at $\pm 54^{\circ}$ to the axis. The bucking coil manifests the same bevel for symmetry, not for mechanical clearance reasons.

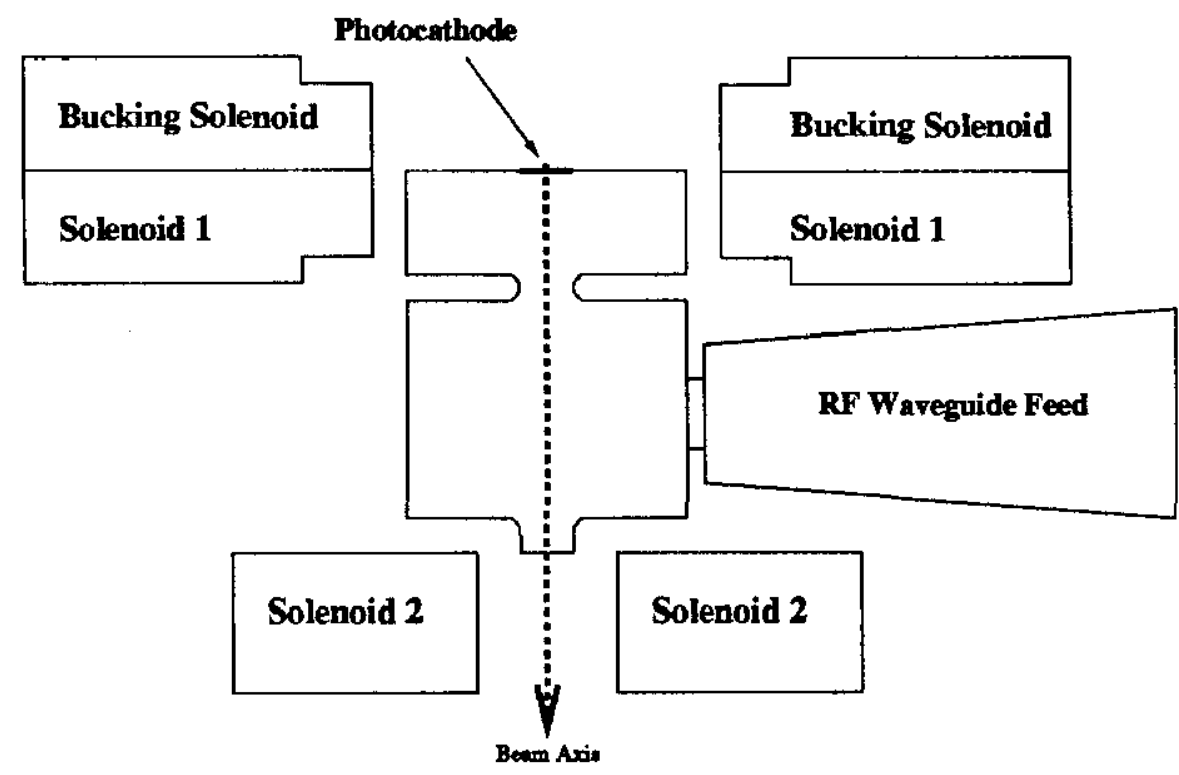

Figure 1: Sketch showing location of focussing and bucking solenoids

The first focussing solenoid and bucking coil have opposing fields, and geometries determined mostly by the space available between the laser ports, the gun exterior, and the cathode plane. The third solenoid follows immediately after the full cell of the gun and has no such space constraints. The solenoid was designed to provide sufficient on-axis focussing strength to allow emittance compensation of beams with normalized energies up to $\gamma=9.0$ on exit from the gun. Table 2 below specifies the electrical characteristics of the first focussing solenoid and the bucking solenoid. The peak field is specified with the bucking solenoid set to cancel the magnetic field at the cathode.

A short section is introduced after the photoinjector to allow the positioning of a six way cross for diagnostics, a vacuum gate valve, and a short drift, to allow the emittance compensation the required time to act before accelerating the beam further, thus "freezing out" the space charge forces.

As the photoinjector is optimized to deliver the required transverse beam quality with reasonable RF power, the lower accelerating gradient will require a longer bunch length to reduce the space charge emittance growth during the relatively longer acceleration time. As a result, compression of the beam must be undertaken once the beam reaches moderate 


\begin{tabular}{|l|c|c|}
\hline \hline Parameter & Symbol & Value \\
\hline \hline Maximum on axis magnetic field & $B_{z}^{\max }$ & $1415 \mathrm{Gauss}$ \\
\hline Maximum excitation current density & $J_{\max }$ & $0.8 \mathrm{kA} / \mathrm{cm}^{2}$ \\
\hline Number of windings & $N$ & 187 \\
\hline Total coil resistance & $R_{c}$ & $158 \mathrm{~m} \Omega$ \\
\hline Voltage drop at peak field & $V_{s}$ & $30.6 \mathrm{Volts}$ \\
\hline Power dissipation at peak field & $P_{\text {diss }}$ & $5.9 \mathrm{~kW}$ \\
\hline Physical Dimensions & \multicolumn{3}{|l|}{} \\
\hline Bore & $r_{i}$ & $12.0 \mathrm{~cm}$ \\
\hline Outer radius & $r_{o}$ & $30.0 \mathrm{~cm}$ \\
\hline Length & $L$ & $10.3 \mathrm{~cm}$ \\
\hline \hline
\end{tabular}

Table 2: Electrical characteristics of the first focussing and bucking solenoids

energy. Magnetic compression requires that the beam pass through a dispersive optical element, making use of a linear energy-phase correlation to reduce the bunch length. Space charges forces will degrade all three emittances during compression, resulting in poor beam quality if compression proceeds for too long or at too low a beam energy. It is therefore optimal to compress at the highest energy possible (thereby reducing the space charge forces) that beamline space allows. The TTF experimental area has rather limited space, motivating the choice to place a magnetic compression chicane at a lower energy ( $20 \mathrm{MeV})$. The proposed beamline is depicted in schematic form in figure 2 below. Detailed simulations

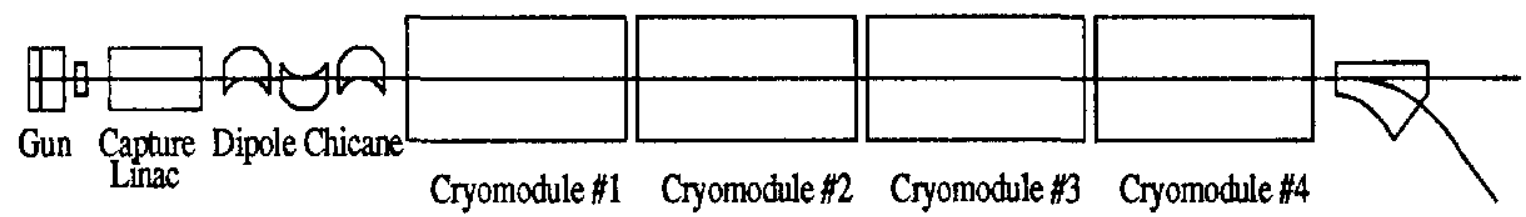

Figure 2: Layout of beamline components for the Tesla Test Facility

of the photoinjector performance were completed using a modified version of PARMELA that accepts field maps for both RF cavity fields and static solenoid fields. The modified code also calculates the effects due to image charges on a metallic photocathode, and has several diagnostics tailored specifically to reveal the underlying phase space dynamics of emittance compensation.

Compression is estimated using the longitudinal emittance of the beam and assuming an ideal linear transformation on the phase space to produce the compressed bunch. Emittance growth resulting from compression is estimated from Carlsten's analysis [5], assuming a short bunch with a radially uniform charge distribution:

$$
\epsilon_{N}=\frac{I S G}{4 I_{A} \beta^{2} \gamma^{2}}
$$

where I is the peak compressed current, $S$ is the path length over which the compressor 
dipole fields act on the beam, $\mathrm{G}$ is a geometric factor between 0.2 and 0.5 , and the Alfven current $I_{A}=4 \pi \epsilon_{o} m_{e} c^{3} / e$.

Table 3 below details the predicted performance of the photoinjector setup. Emittance values quoted are one sigma normalized values enclosing $100 \%$ of the stated bunch charge. (In each case, this represents $80 \%$ of the total initially launched charge, with the $20 \%$ excess "guard charge" being collimated away) Two different operating scenarios were examined: high bunch charge ( $8 \mathrm{nC} /$ bunch) operation for testing of HOM energy deposition in superconducting RF linac structures, and low bunch charge $(1 \mathrm{nC} / \mathrm{bunch})$ operation for injecting into a free electron laser (FEL). Significant effort was devoted to optimizing the $8 \mathrm{nC}$ scenario, both for highest beam quality, and for lowest possible RF power consumption. The low charge $(1 \mathrm{nC})$ case was derived by scaling the bunch radius and length to preserve the bunch core charge density, thereby allowing the emittance compensating lens configuration and strength to remain essentially unchanged. Although the $1 \mathrm{nC}$ case outlined below has not been fully optimized, good beam quality $(\epsilon=2.5 \pi \mathrm{mm}-\mathrm{mr})$ and moderately high peak current ( 80 Amperes) are present before compression, which contributes approximately 0.52 $\pi \mathrm{mm}$-mr to the transverse emittance while raising the peak current to 120 Amperes.

\begin{tabular}{|lccc|}
\hline Parameter & Symbol & Predicted Value & Predicted Value \\
\hline \hline & & HOM ANALYSIS & FEL \\
\hline Before Compression & $Q_{b}$ & $8 \mathrm{nC}=5 \times 10^{10} e^{-}$ & $1 \mathrm{nC}=6 \times 10^{9} e^{-}$ \\
\hline Bunch Charge & $\Gamma_{t}$ & $28 \mathrm{ps}$ & $13.5 \mathrm{ps}$ \\
\hline Laser pulse length FWHM & $\phi_{o}$ & $45^{\circ}$ & $35^{\circ}$ \\
\hline Launch Phase (w.r.t. $\left.\bar{E}_{z}=0\right)$ & $r_{o}$ & $3.0 \mathrm{~mm}$ & $1.4 \mathrm{~mm}$ \\
\hline Beam radius at cathode & $\gamma_{1}$ & 12.3 & 12.3 \\
\hline Post-Gun Gamma & $\gamma_{f}$ & 41.5 & 41.5 \\
\hline Post-Linac Gamma & $\gamma_{f}$ & $15 \pi \mathrm{mm}-\mathrm{mr}$ & $2.5 \pi \mathrm{mm}-\mathrm{mr}$ \\
\hline Horizontal Emittance & $\epsilon_{x}$ & $15 \pi \mathrm{mm}-\mathrm{mr}$ & $2.5 \pi \mathrm{mm}-\mathrm{mr}$ \\
\hline Vertical Emittance & $\epsilon_{y}$ & $1500 \mathrm{deg}-\mathrm{keV}$ & $1200 \mathrm{deg}-\mathrm{keV}$ \\
\hline Longitudinal Emittance & $\epsilon_{z}$ & $7.93 \%$ & $1.2 \%$ \\
\hline Momentum Spread & $\delta p / p_{o}$ & $3.1 \mathrm{~mm}$ & $1.27 \mathrm{~mm}$ \\
\hline Bunch Length & $\sigma_{b}$ & $386 \mathrm{Amperes}$ & $80 \mathrm{Amperes}$ \\
\hline Peak Current & $I_{p}$ & & \\
\hline After Compression & & & $3.02 \pi \mathrm{mm}-\mathrm{mr}$ \\
\hline Horizontal Emittance & $\epsilon_{x}$ & $19.4 \pi \mathrm{mm}-\mathrm{mr}$ & $1 \mathrm{~mm}$ \\
\hline Vertical Emittance & $\epsilon_{y}$ & $19.4 \pi \mathrm{mm}-\mathrm{mr}$ & $3.02 \pi \mathrm{mm}-\mathrm{mr}$ \\
\hline Bunch Length & $\sigma_{b}$ & $1 \mathrm{~mm}$ & $120 \mathrm{Amperes}$ \\
\hline Peak Current & $I_{p}$ & $958 \mathrm{Amperes}$ & \\
\hline \hline
\end{tabular}

Table 3: Predicted performance of the Photoinjector

Shown in figure 3 below are the evolution of the transverse beam emittance, bunch length, and bunch radius throughout the injector for the high bunch charge case. Four emittance traces are shown, representing the FWHM one-sigma normalized emittances of 

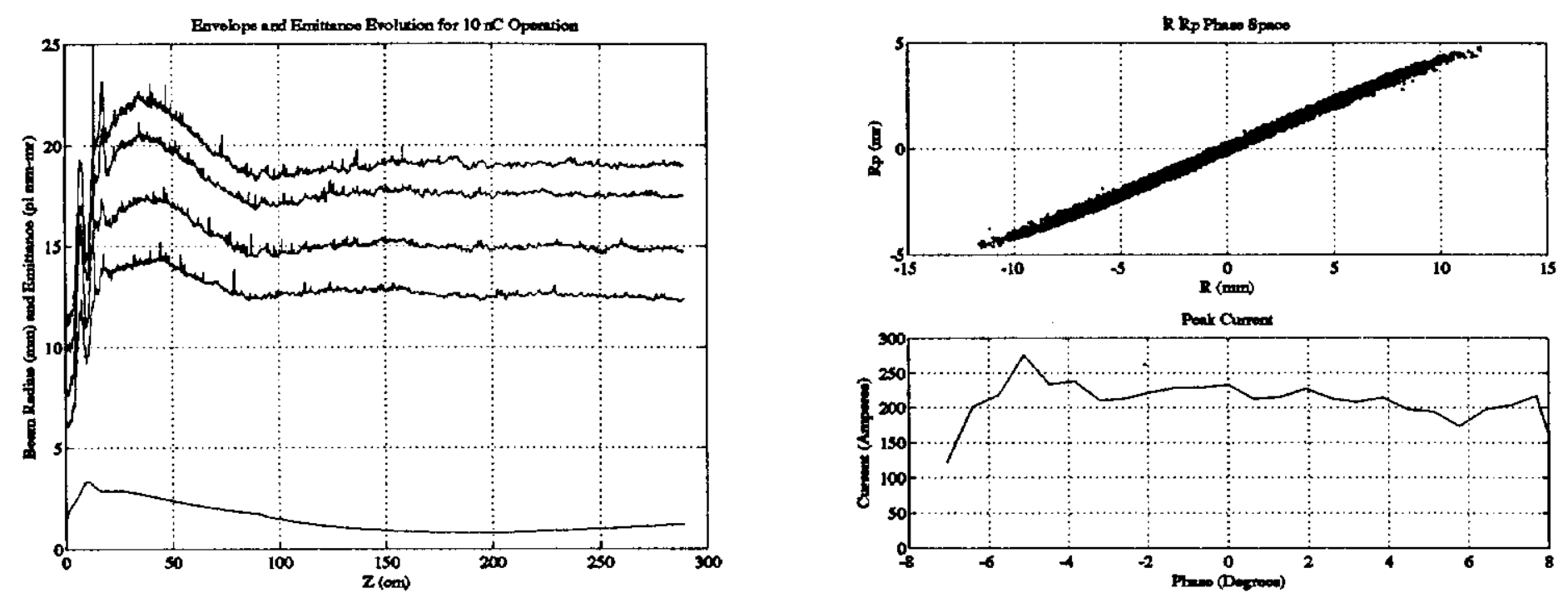

Figure 3: Emittance and envelope evolution for $10 \mathrm{nC}$ operation, final phase space plots

$95 \%, 90 \%, 80 \%$, and $70 \%$ of the bunch particles. (viz. An $80 \%$ emittance represents the emittance the bunch would have if the outermost $20 \%$ of the beam particles were collimated away) The onset of emittance compensation is clearly visible in the decrease of all emittances after the solenoid focussing kick. Transverse and longitudinal phase space plots at the end of the injector beamline (prior to compression) are shown at right. Figure 4 shows analogous plots for operation at $1 \mathrm{nC}$ bunch charge. For reference, the gun exit is located at $z=20.7$ $\mathrm{cm}$, is followed by a drift up to $z=96.2 \mathrm{~cm}$ where the beam enters a 9 cell linac extending to $z=200$. $\mathrm{cm}$, where the beam is again in a drift.

\section{RF Design of the Photoinjector}

Simplicity, high shunt impedance, and the ability to accommodate an externally mounted focussing solenoid close to the cathode region motivated the choice of a 1.5 cell $T M_{010, \pi}$ mode structure. The need to induce a strong on-axis magnetic field made the use of a superconducting cavity problematic, and the need for a high accelerating gradient eliminated the choice of a superconducting gun altogether. The Brookhaven/Grumman [Gun I] S-band photoinjector [10] was taken as a starting point for the design of the TTF injector; the resonant frequency, waveguide coupling, intercavity coupling, and longitudinal exit aperture profile were modified to yield an L-band structure with good shunt impedance and field balance characteristics.

Placement of the focussing solenoid around the first half cell of the gun completely occludes all reasonable locations for an RF coupling slot, requiring power for the half cell to be coupled in either magnetically through a series of coupling slots placed at the maximum of the azimuthal magnetic field, or electrically by widening the iris. Slot coupling is known to excite higher order azimuthal spatial harmonics in the RF field causing unwanted nonlinear 

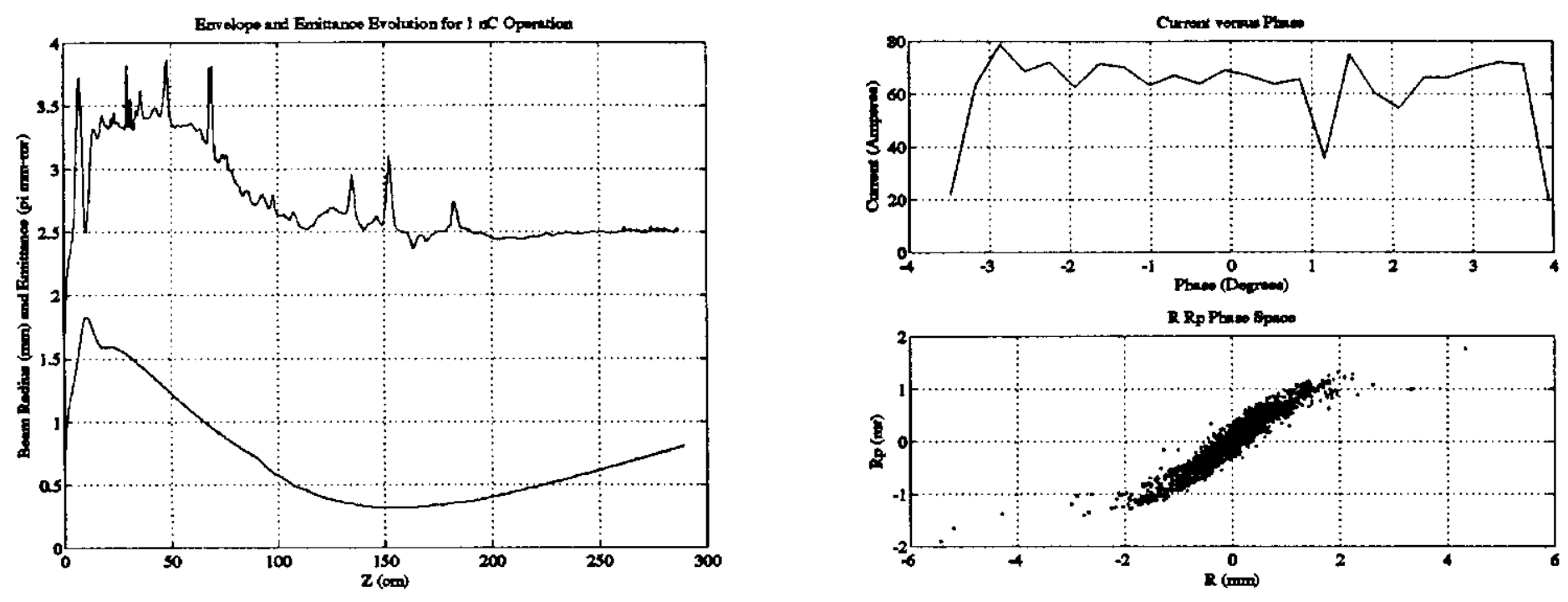

Figure 4: Emittance and envelope evolution for $1 \mathrm{nC}$ operation, final phase space plots

RF transverse emittance growth. Widening the iris for improved group velocity lowers the shunt impedance and affects the radial spatial harmonics (flattening the near-axis radial variation of $E_{z}$ as a benefit) and reduces the strength of the higher order longitudinal spatial harmonics. This somewhat unusual coupling scheme has already been employed successfully in a photoinjector for a free electron laser [14, 13].

Although RF power efficiency was of prime concern in designing the photoinjector, the standard shunt-impedance increasing procedure of decreasing the gap length with the addition of "reentrant noses" on the entrance and exit irises was not undertaken. Although such a geometric modification can appreciably improve the power efficiency of an RF structure, it does so at the expense of significantly enhancing the nonlinear components of the accelerating field. As the rms bunch radius for the high-charge case is significant $(7 \mathrm{~mm}$ at the maximum, $10 \mathrm{~cm}$ from the photocathode), nonlinear $\mathrm{RF}$ emittance growth in such a cavity would be unacceptably large. Also, the large accelerating gradient (twice the Kilpatrick threshold) makes an RF structure with a low peak-field to accelerating-field ratio especially desirable, making the addition of any geometric disturbances in the high electric field region of the cavity undesirable.

An elongated half cell was chosen to provide improved beam divergence control (A small amount of RF focussing occurs in the region right off the cathode as a result of the lengthening) and additional time to start the solenoidal focussing kick to initiate the emittance compensation before the time-dependent kick of the first iris becomes appreciable. After some optimization, a half cell length equal to $\frac{5}{4} \frac{\lambda_{R F}}{4}$ was chosen. To ameliorate further higher spatial harmonic pollution of the accelerating mode, the full cell has a length that is exactly $\frac{\lambda_{R F}}{2}$.

The iris diameter between the full cell, where RF power is coupled in, and the half cell was chosen to yield strong enough coupling that the longitudinal position of the photocathode could be used as a frequency tuning mechanism without causing a substantial shift 
in the field balance between the two cells. Various field balance options were considered, motivated by the possibility of using RF focussing at the first iris on the one hand, and by the possibility of improved longitudinal phase space linearity (and thus compressibility) on the other. A field imbalance between the half and full cell could be made to enhance the time dependent focussing kick centered at the first iris, but was found to significantly interfere with emittance compensation, degrading final beam quality, and was not pursued further. Thus a balanced $\left(E_{z, \max }^{\text {halfcell }}=E_{z, \max }^{\text {fullcell}}\right)$ field profile was chosen. To ensure the field balance, the mode separation was chosen to be approximately $2.5 \mathrm{MHz}$ (40 times the $-3 \mathrm{~dB}$ cavity bandwidth), implying a coupling constant of $\gamma=0.19 \%$. Assuming the half cell field to be $E_{1}=45 \mathrm{MV} / \mathrm{m}$, the full cell field $E_{2}=35 \mathrm{MV} / \mathrm{m}$, the stored energy $U=10.5 \mathrm{~J}$, the iris thickness to be $d=1.5 \mathrm{~cm}$, and the free space wavelength $\lambda=23.061 \mathrm{~cm}$, the electrical coupling iris radius needs to be [6]:

$$
r_{o}=\left[\frac{3 \gamma U}{2 \epsilon_{o} E_{1} E_{2} e^{-\alpha d}}\right]^{\frac{1}{3}} \approx 2.0 \mathrm{~cm}
$$

where $\alpha=k_{o} \sqrt{\left(\lambda / \lambda_{c}\right)^{2}-1}$ is the attenuation length for the $T E_{11}$ mode. Simulation of the cavities using the Superfish code yields $\gamma=0.189$ for an iris radius of $2.0 \mathrm{~cm}$, in good agreement with prediction. As the hole is not uniform in radius, (rather the edges are rounded to prevent field line concentration) the coupling constant for the simulated and actual photoinjector will be somewhat higher than equation 2 predicts.

The effects of beam loading also bear directly on the choice of coupling strength, as loading in each cell of the photoinjector is different, leaving the fields slightly imbalanced (ie the zero mode is weakly excited) after the bunch has passed. The coupling strength will influence the recovery time of the photoinjector, which must be significantly less than the time between bunches. In view of the short time between successive bunches, and the large number of bunches per pulse, a simple examination of the RF transient response of the photoinjector was made. A lumped circuit model, shown in figure 5, was used to analyze the fill rates of the two cavities, and verify that the coupling was adequate. The coupled

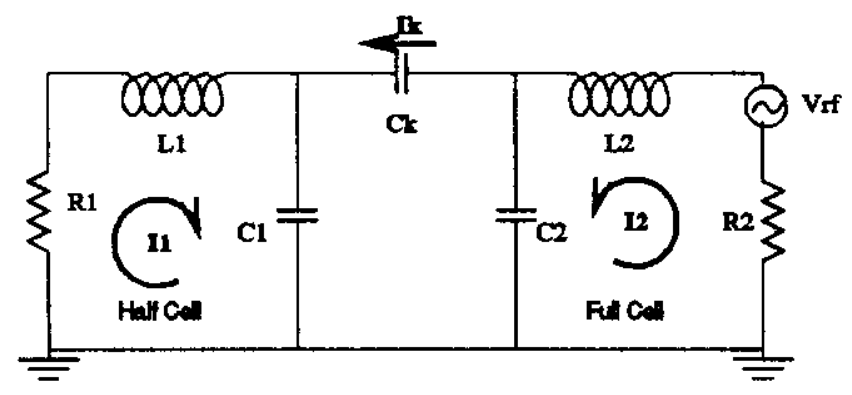

Figure 5: Lumped circuit model of two cell electrically coupled $\pi$-mode structure

differential equations governing the currents $I_{1}(t), I_{2}(t)$ and $I_{k}(t)$ may be solved in the weak coupling approximation to yield the fill times for the two cavities:

$$
\tau_{1} \approx \tau_{2} \approx \frac{2 L}{R}=\frac{2 Q}{\omega} \approx 2.91 \mu s
$$


where the lumped circuit component values were estimated using the loaded $Q$, structure impedance $\mathrm{Z}$, (not $Z T^{2}$ ), and the resonant frequency of the individual cavities as calculated by Superfish.

Direct numerical integration of the exact equations using a 4th order Runge-Kutta algorithm generated figure 6 . The mode amplitudes are shown on the left as a function of time for the first $25 \mu \mathrm{s}$, and in a Lissajous plot on the right, showing the relative phase of the two cavities as the $\pi$-mode builds up. The beating of the zero and pi modes is plainly visible during the fill in both mode plots, but as the zero mode is undriven and damps exponentially, there is little modulation of the mode amplitudes once the cavities are filled. More significant, however, is that the fill times for the two cavities are indeed comparable, despite the small coupling constant. This can be explained by noting that the $Q$ of the cavities is much larger than the inverse of the coupling constant.

Beam loading in the half cell is expected to be:

$$
P_{b e a m}=\int E_{z} d z \cdot \frac{N_{b} Q_{b}}{\tau_{R F}} \approx 18.5 k W
$$

which is to be compared with the power dissipation on the walls of the half cell:

$$
P_{d i s s, H C}=\frac{R_{s}}{2} \int H_{\phi}^{2} d \mathcal{S} \approx \frac{R_{s}}{2} \frac{\epsilon_{o}}{\mu_{o}} \frac{k^{2}}{k_{r}^{2}} E_{o}^{2} J_{1}^{2}\left(k_{01}\right) 2 \pi R \frac{L_{z}}{2} \approx 0.94 M W
$$

for $E_{0}=35 \mathrm{MV} / \mathrm{m}$, or less than $2 \%$. Since the entire cavity fills in $3 \tau_{R F}=8.7 \mu \mathrm{sec}$, recovery from beam loading will be well within the $1 \mu \mathrm{sec}$ requirement. The aformentioned pi-mode iris-coupled structure [14] has much more severe beam loading (2.3 $\mathrm{MW}$, versus a wall loss of only $0.7 \mathrm{MW}$ ) and a lower intercavity coupling constant. The input coupler for the
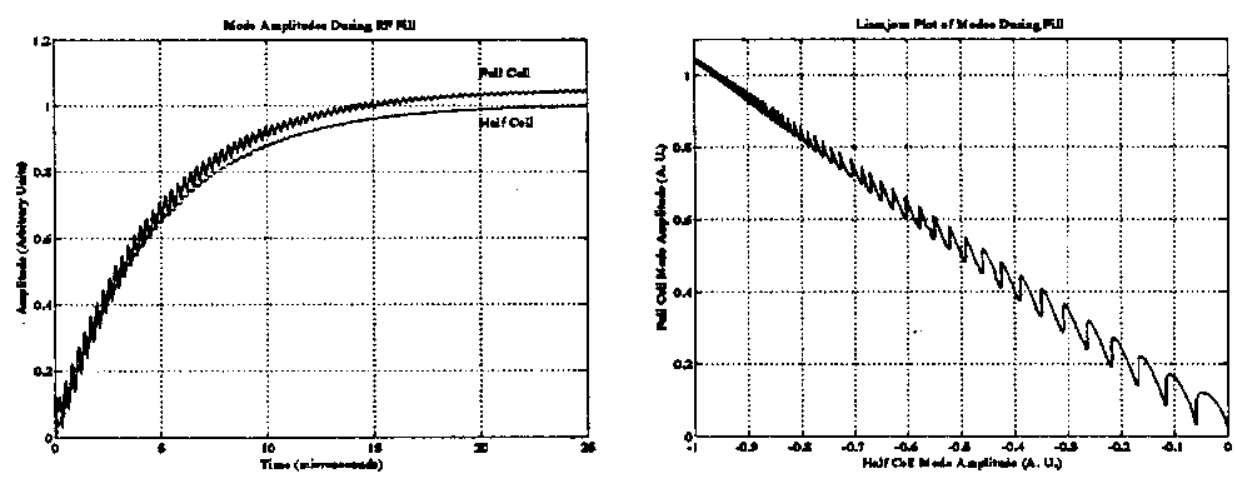

Figure 6: RF Fill of Half and Full Cell

photoinjector was simulated using Hewlett Packasd's High Frequency Structure Simulator 
(HFSS), which is a fully three-dimensional finite element frequency domain electromagnetic code. As a guide for choosing the dimensions of the coupling slot, Gao's expression [7] for the coupling constant, $\beta$, derived using Bethe's formalism for computing the perturbation of cavity fields due to apertures [2], was employed, with the dissipated power $P_{o}$ taken to be the total power dissipated in both cavities:

$$
\beta=\frac{\pi Z_{o} k_{o}}{9} \frac{\Gamma_{10}}{W_{w g} H_{w g}} \frac{e_{o}^{4} e^{-2 \alpha \delta} l_{1}^{6}}{\left(\mathcal{K}\left(e_{o}\right)-\mathcal{E}\left(e_{o}\right)\right)^{2}} \frac{H_{\phi}^{2}}{P_{o}}
$$

With the impedance of free space $Z_{0}=120 \pi$, the free space RF wavenumber $k_{0}=\frac{2 \pi}{\lambda_{0}}$, the waveguide propagation constant $\Gamma_{10}=k_{o} \sqrt{1-(\lambda / 2 a)^{2}}$, the aperture mode attenuation constant $\alpha=k_{o} \sqrt{\left(\left(\frac{\lambda}{\lambda_{c}}\right)^{2}-1\right)}$ with cutoff wavelength $\lambda_{c}=3.41 \sqrt{l_{1} l_{2}}, \delta$ being the aperture depth, $W_{w g}$ and $H_{w g}$ the width and height of the waveguide, respectively, $H_{\phi}$ the tangential magnetic field strength at the aperture location, and the aperture eccentricity $e_{o}=\sqrt{\left(1-\left(\frac{l_{2}}{l_{1}}\right)^{2}\right)}$. Figure 7 below shows the characteristically rapid variation of $\beta$ with aperture length. The length of the aperture in the $z$-direction is held fixed at $2.0 \mathrm{~cm}$ for both plots. In view of the very light beam loading $\left(18.5 \mathrm{~kW}\right.$ at $\left.E_{o}=35 \mathrm{MV} / \mathrm{m}\right)$, the unloaded
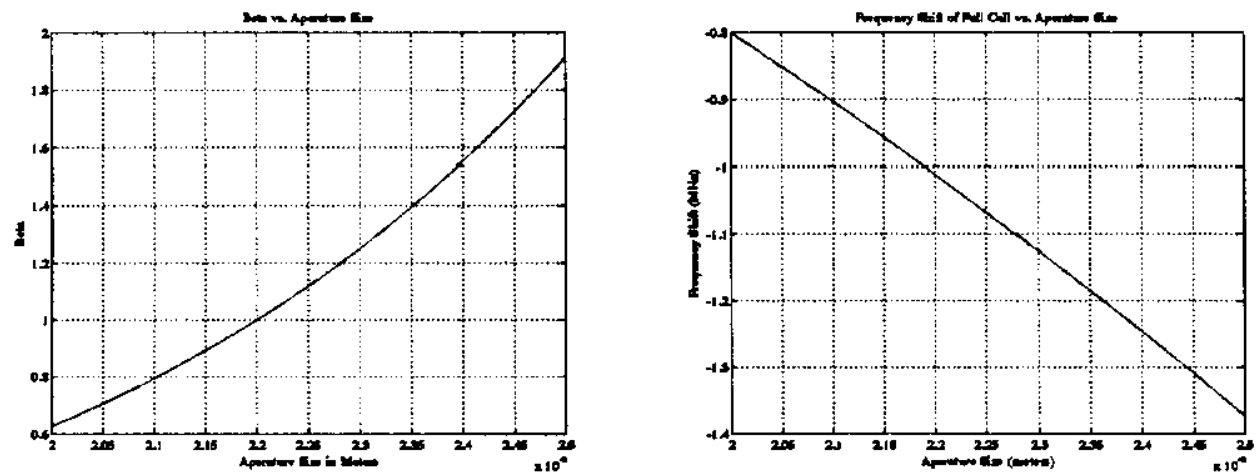

Figure 7: Coupling $\beta$ and cavity frequency shift (in $\mathrm{MHz}$ ) versus aperture length (in $\mathrm{cm}$ ).

cavity coupling coefficient need not be adjusted much to assure critical coupling with the beam present. The adjusted unloaded cavity coupling coefficient should be $\beta_{o}=1+\frac{P_{\text {eam }}}{P_{\text {cav }}}$ or approximately 1.02 for this case. In addition, this effect can be controlled by feed-forward on the rf amplitude.

The opening of the coupling slot on the outer wall lowers the cavity frequency because of the effective increase in the cavity volume in an $\mathrm{H}$-field dominated region. We examined the possibility of compensating for the frequency depression by a simple technique. The waveguide end may be pushed into the volume of the cavity, producing a flat region that not only decreases the volume of the cavity, thereby raising the frequency, but makes the thickness of the waveguide coupling slot more uniform. The two frequency perturbations can be made to cancel by an astute choice of the waveguide's penetration distance into the cavity, once the required dimensions of the coupling slot are known. The intrusion depth for this RF structure was calculated and found to be too small to warrant the added machining 
complication. Instead, the radius of the full cell has been adjusted to provide the required equal and opposite frequency shift.

Lastly, the waveguide taper, required to allow space for the first focussing solenoid (see figure 1) and to match the WR650 waveguide to the cavity, was chosen to be a standard $\lambda / 4$ stepped transformer. Simulations with HFSS indicate that suppressing reflections by $20 \mathrm{~dB}$ or more is straightforward. The electrical properties of the accelerating structure are summarized in table 4 below.

\begin{tabular}{|l|c|c|}
\hline \hline Parameter & Symbol & Value \\
\hline \hline Operating mode & & $T M_{010, \pi}$ \\
\hline Frequency & $f$ & $1300 \mathrm{MHz}$ \\
\hline Transit time factor & $T$ & 0.73127 \\
\hline Structure quality factor & $Q$ & 23700 \\
\hline Structure fill time & $\tau_{R F}$ & $2.90 \mu \mathrm{s}$ \\
\hline Structure Shunt Impedance & $Z_{s}$ & $13.82 \mathrm{M} \Omega$ \\
\hline Effective Shunt Impedance & $Z T^{2}$ & $27.92 \mathrm{M} \Omega / \mathrm{m}$ \\
\hline Peak-to-accel field ratio & $E_{p k} / E_{a c c}$ & 1.870 \\
\hline Power diss at $E_{\text {acc }}=50 \mathrm{MV} / \mathrm{m}$ & $P_{\text {diss }}$ & $4.5 \mathrm{MW}$ \\
\hline Average Power diss at $E_{a c c}=50 \mathrm{MV} / \mathrm{m}$ & $P_{a v e}$ & $45.0 \mathrm{~kW}$ \\
\hline \hline
\end{tabular}

Table 4: Electrical Properties of the Photoinjector

As with any asymmetrically iris-coupled structure, the electrical center of the fields is shifted towards the driving iris. A least squares fit of the near-axis data from HFSS reveals that the displacement of the field maximum is $\leq 0.39 \mathrm{~mm}$ from the geometric center of the cavity. Such a small shift (which is present only in the full cell, owing to the RF coupling scheme used) is not enough to warrant a cavity geometry alteration to compensate.

Run time tuning of the photoinjector will be accomplished with the aid of four largely separate controls. Gross frequency tuning of the photoinjector as a whole will be accomplished by regulating the cooling water temperature. Thermal analysis of a similar L-band structure dissipating much higher average power $(150 \mathrm{~kW}$, versus $45.0 \mathrm{~kW}$ for the present case) found no serious difficulties in providing adequate cooling[11]. Indeed, an S-band structure sustaining the same duty cycle $(1 \%)$ but higher accelerating gradient (by a factor of 2), and thus substantially higher dissipated power density (by a factor of 20), has been designed and successfully operated at Brookhaven National Lab [10].

For an L-band copper structure, the frequency change per degree Kelvin is approximately $\Delta f / \Delta T=-\left(k_{01} \cdot c \cdot \kappa_{T}\right) /(2 \pi R) \approx 21.8 \mathrm{kHz} / \mathrm{K}$, with the thermal coefficient of expansion $\kappa_{T}=16.92 \times 10^{-6} / \mathrm{K}$ for copper. Tuning of the half and full cells is accomplished by means of a paddle-style tuner in each cell, allowing individual frequency control (details of the paddle design may be found in Appendix A). Lastly, the photocathode will be mounted on a micrometer, allowing fine longitudinal positioning of the cathode, and thus the resonant frequency of the half cell. Assuming the cathode and paddle actuators provide a linear positioning precision of 20 microns and an angular positioning precision of 5 degrees (paddles 
only), the tuning sensitivities will be $-17.94 \mathrm{kHz} / 20$ micron, $28.0 \mathrm{kHz} / 20 \mathrm{micron}$, and 3.1 $\mathrm{kHz} / 5$ degrees, respectively.

\section{Diagnostics and Instrumentation}

The layout of the Argonne National Laboratory photoinjector test setup is shown in Figure 8 below. Instrumentation of the beamline has been chosen to permit exploration both of the injector performance in general, and to allow direct observation of emittance compensation. A new approach to emittance measurement has been proposed to explore the

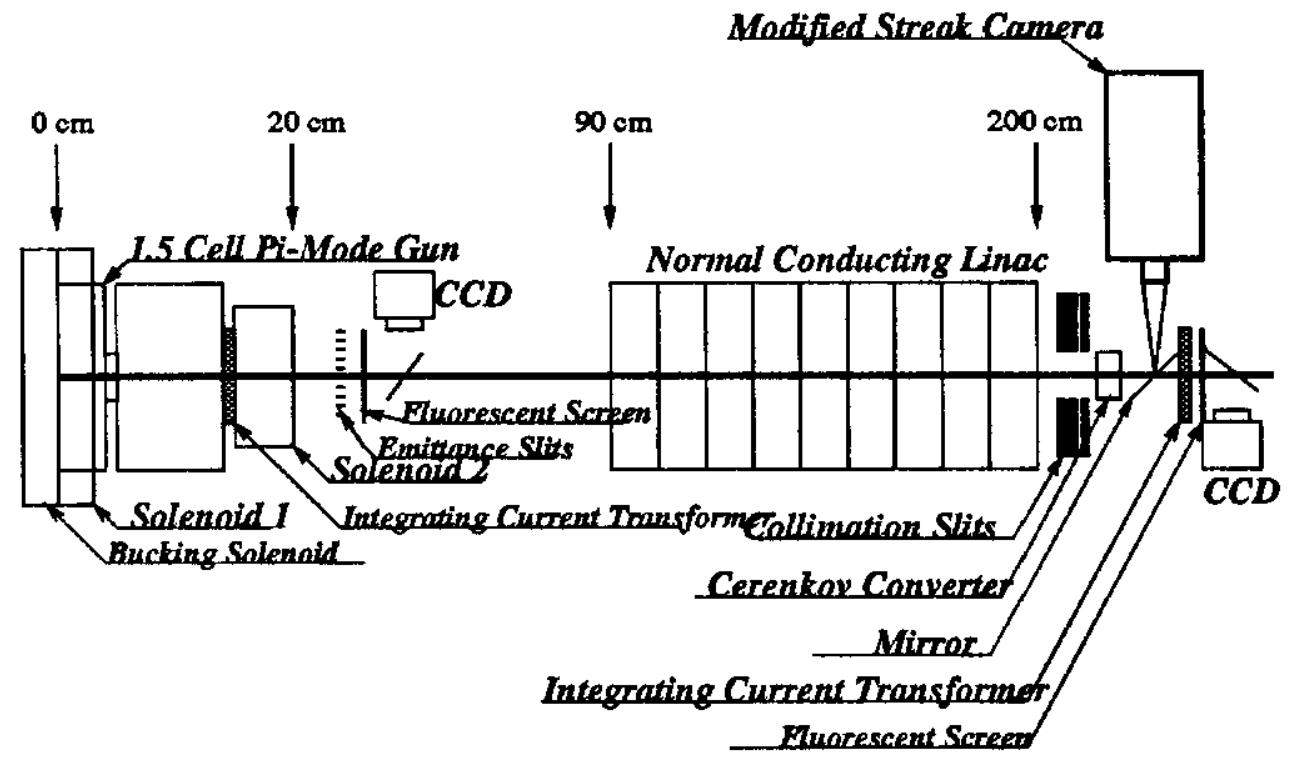

Figure 8: Layout of beamline components for testing the photoinjector at A.N.L.

physical basis for emittance compensation. A time-resolved variation of a slit emittance measurement technique (similar in principle to the pepper pot) employed at UCLA[12] will be used, providing emittance measurement in one transverse plane as a function of longitudinal position within the beam. While prior time-resolved measurements of electron beam emittance on the nanosecond time scale at Los Alamos [8], and of three-dimensional spatial distribution at LEP [1] have been successfully undertaken, measurements with sufficient resolution (picosecond or better) to observe the emittance compensation process have not. Figure 9 provides a schematic of the proposed measurement. A detailed analysis of the apparatus will appear in the near future, but a brief description is included here for completeness.

The space charge dominated beam is brought to a non-ballistic waist (ie particles do not cross the axis) and collimated into several emittance dominated beamlets by a slit emittance mask. The beamlets retain the transverse temperature of the original beam, but at such reduced charge that space charge forces within the individual beamlets contribute negligibly to their momentum spread. The beamlets then drift several meters to allow the correlated transverse momentum time to impart a measurable transverse distance offset, and are passed 


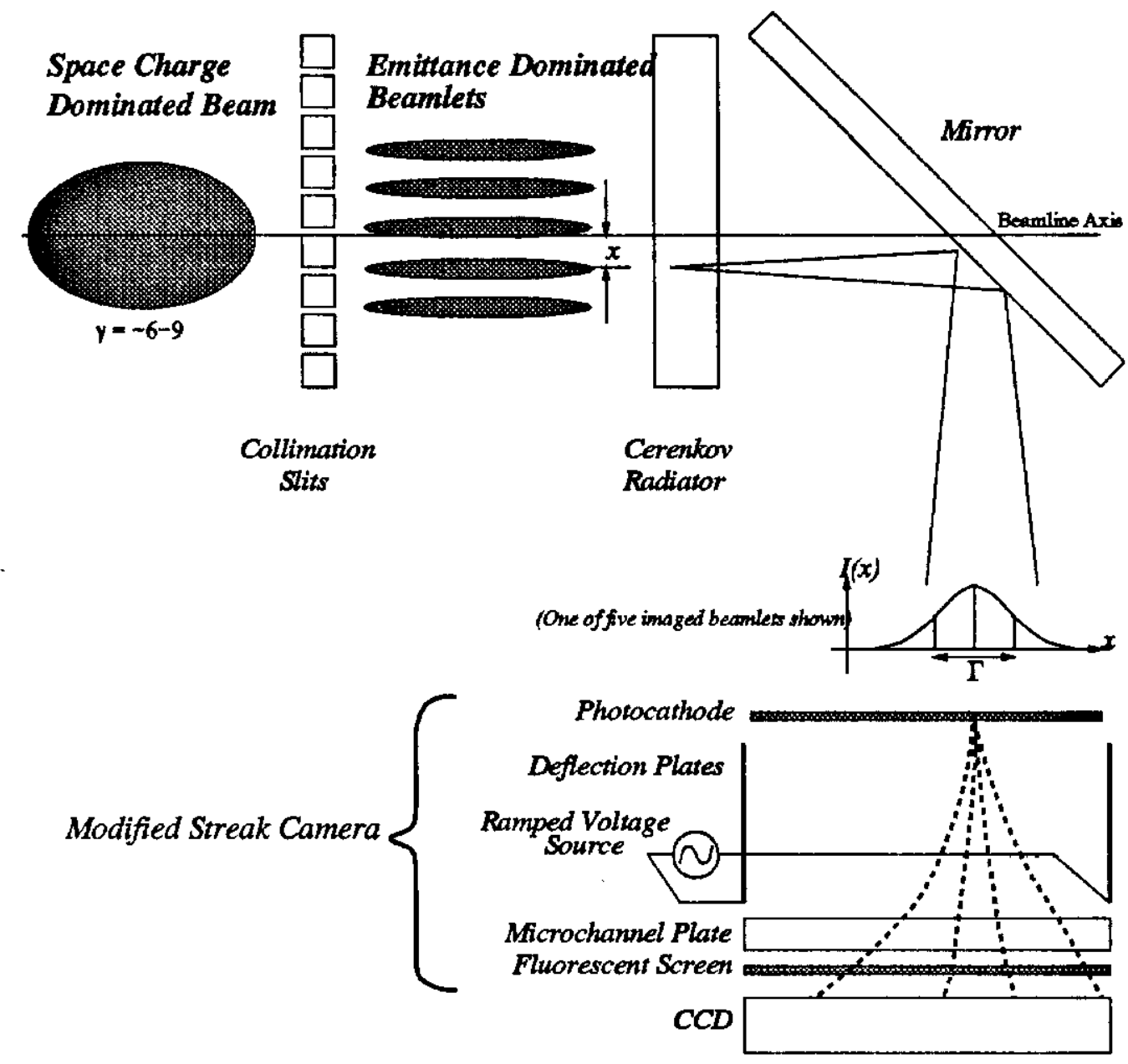

Figure 9: Schematic of time resolving emittance measurement apparatus

through a Cerenkov radiator to produce an optical signal that can be extracted from the beamline. The light is focussed (optics not shown) onto the photocathode of a streak camera, and the streaked image recorded with a high resolution CCD camera. The spread of the light from each beamlet may be analyzed to unfold the contribution due to the transverse temperature of the beam from the natural spread angle of the Cerenkov radiation. The centroid of the beamlets at the radiator provides the centroid of the transverse momentum spread, while the transverse position centroid is known immediately from the separation of the collimator slits. From these data the transverse phase space of the beam may be reconstructed as a function of longitudinal position within the beam. The slit separations are chosen to ensure that the light from adjacent beamlets does not overlap at the CCD camera.

The measurement will be made at two locations, once at the photoinjector exit, and again at the exit of the linac, allowing comparison of the beam's slice emittances immediately after the focussing kick, and shortly after the emittance compensation minimum has occured.

With this diagnostic, investigation of the effectiveness of emittance compensation will be 
explored as a function of compensating lens position and strength by adjusting the current sum and the current ratio, respectively, of the two focussing solenoids depicted in figure 1 above. With information about the actual slice emittances (viz. the emittance of a subset of the beam electrons between $z$ and $z+\Delta z$ ) and the orientation of the slice emittance ellipses before and after compensation, detailed study of emittance compensation will be possible for the first time.

In addition, two integrating current transformers will be mounted, one immediately downstream of the photoinjector to non-destructively monitor bunch charge at emission, and another after the collimators downstream of the first linac section to monitor final beam charge. Ordinary fluorescent screens, fitted with CCD cameras, used with the emittance slit mask, will provide ensemble emittance measurements as a cross check for the time resolving emittance measurement. A dipole spectrometer (not shown) will be mounted at the end of the beamline for beam momentum analysis.

The RF and laser systems at Argonne National Laboratory Wakefield Accelerator Facility are under computer control through a combination of LabView and custom $\mathrm{Tcl} / \mathrm{Tk}$ based software. The TTF photoinjector will make use of the existing RF and laser systems, adding a separate LabView-based control system to run the diagnostics. A number of LabView "virtual instruments" (VIs) have been developed to operate similar equipment at the UCLA Particle Beam Physics Laboratory, and will be transferred to the TTF photoinjector with minimal additional programming effort. The time-resolved emittance diagnostic will require the development of custom software to process the streak data.

\section{Timetable}

A timetable indicating the expected start and end dates of the three phases of the photoinjector design program is shown in figure 10 below. Annual boundaries shown are those of the fiscal year. Work begun on the hardware development at the time of this writing includes emit tance measurement system development, and construction of a cold test model of the 1.5 cell gun.

\section{Conclusion}

An RF and beam optics design for a Tesla Test Facility compatible electron photoinjector has been developed and presented here in brief. Further work to improve the beam dynamical aspects of the photoinjector assembly will continue, focussing on improvements in beam compressibility and a careful examination of the photoinjector's operation at reduced charge, of particular interest for the proposed FEL user facility that may evolve from the TTF.

\section{Acknowledgements}

The authors wish to thank Bruce Carlsten, Patrick O'Shea and Richard Sheffield for helpful discussions of the emittance compensation technique and the commissioning of the AFEL photoinjector, Ralph Pasquinelli and Bill Miller for informative discussions on the details 
Timeline for Photoinjector Development

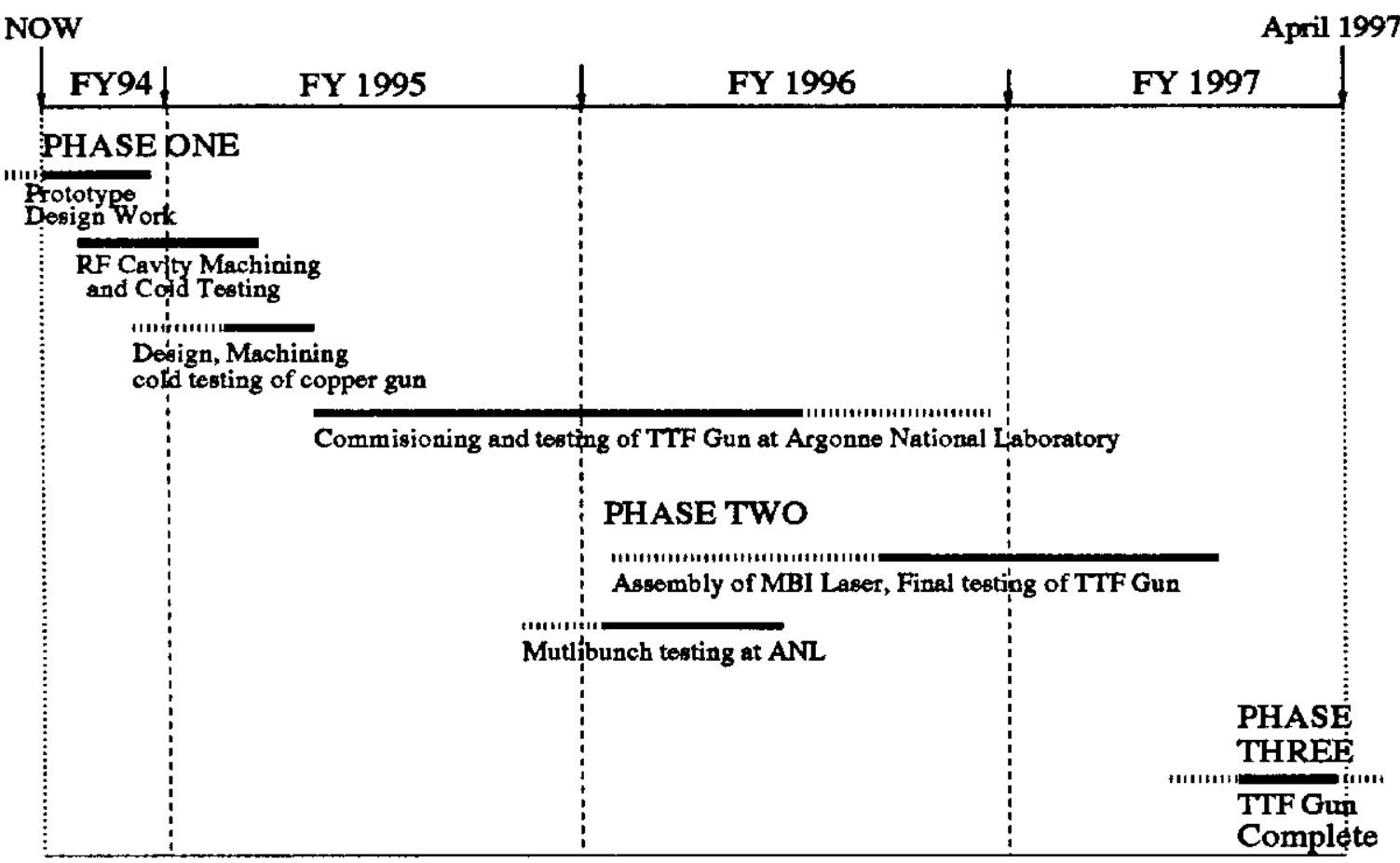

Figure 10: Projected timetable for TTF photoinjector design and commissioning activities

of the RF design, Vinod Bharadwaj for his continuing support and encouragement, and Christian Travier for frank discussion of the design and commissioning of the CANDELA photoinjector.

\section{References}

[1] G. Baribaud, C. Bovet, R. Jung, M. Placjdi, E. Rossa, Y. Solberg, Proc. of the $X V^{\text {th }}$ Int. Conf, on High Energy Accel., p.212-4.

[2] H. A. Bethe, The Physical Review, 66, Nos. 6 and 7, p.163-82 (1944).

[3] V. K. Bharadwaj, private communication, May 1994.

[4] B. E. Carlsten, NIM A285, p.313-9, (1989).

[5] B. E. Carlsten, "Emittance Growth of Bunched Beams in Bends", unpublished paper, (1993).

[6] J. Gao, NIM A311, p.437-43, (1992).

[7] J. Gao, NIM A311, p.431-6, (1992). 
[8] T. Kauppila, R. Carson, D. Moir, and R. Ridlon, Proc. of the 1991 IEEE Conf., San Francisco, p. 2107-9.

[9] K. J. Kim, NIM A275, p201-18, (1989).

[10] I. S. Lehrman, I. A. Birnbaum, M. Cole, S. Z. Fixler, R. L. Hauer, S. Siddiqi, E. Sheedy, I. Ben-Zvi, K. Batchelor, J. C. Gallardo, H. G. Kirk, T. Srinivasan-Rao, and G. D. Warren, "Design of a High-Brightness, High-Duty Factor Photocathode Electron Gun", NIM A318, p.247-53, (1992).

[11] J. B. Rosenzweig, E. R. Colby, G. P. Jackson, and T. Nicol, Proc. of IEEE PAC 1993, Washington D. C.

[12] J. B. Rosenzweig, G. Travish, "Design Considerations for the UCLA PBPL Slit-Based Phase Space Measurement Systems",PBPL internal, Feb. 22, 1994. (Attached as appendix C.)

[13] H. Sakae, M. Takabe, M. Ogoshi, and S. Mandai, NIM A331, p.346-9, (1993).

[14] M. Takabe, Y. Miyauchi, H. Sakae, M. Ogoshi, T. Komatsu and S. Mandai, NIM A318, p.367-71 (1992). 


\section{Appendices}

\subsection{Appendix A: Design of the Tuning Paddles}

Small run-time adjustments to the resonance frequency of each cavity (of order $1 \mathrm{MHz}$ or less) are provided for by means of a tuning "paddle" placed in each cell, depicted schematically below. Axial motion of the paddle provides tuning in the same manner as a plunger

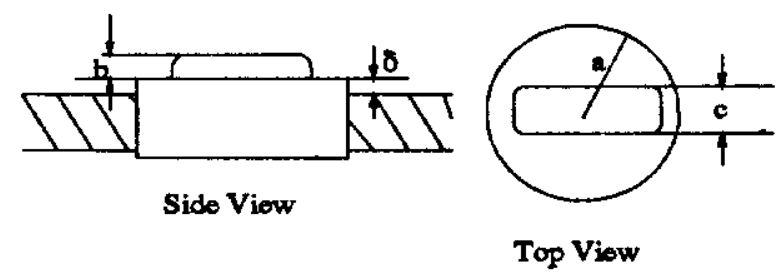

Figure 11: Schematic Drawing of the Tuning Paddle

would, while rotation of the paddle controls whether the axis of the paddle is parallel to the cavity field polarization, thereby sampling the $\cos \left(k_{z} z\right)$ drop off of the fields, or perpendicular, intercepting roughly constant fields, and giving the greatest frequency shift. From Slater's theorem, a perturbation in the geometry of an RF cavity produces a shift in the resonance frequency that is approximately proportional to the amount of electric and magnetic field energy displaced:

$$
\omega^{2}=\omega_{o}^{2}\left(1+\frac{1}{2 U} \int_{\mathcal{V}}\left(\mu_{o} H^{2}-\epsilon_{o} E^{2}\right) d V\right)
$$

with $U$ representing the total stored energy in the cavity, and $\mathcal{V}$ the volume displaced by the perturbing object. The electric and magnetic fields for the lowest space harmonic of the $T M_{010, \pi}$ mode of a pill box cavity (with on axis irises at both ends), with radius $R$ and length $L_{z}$ are:

$$
\begin{aligned}
& E_{r}(\rho, \theta, z)=-E_{o} \frac{k_{z}}{k_{r}} J_{1}\left(k_{01} \rho / R\right) \cos \left(k_{z} z\right) \\
& E_{z}(\rho, \theta, z)=E_{o} J_{o}\left(k_{01} \rho / R\right) \sin \left(k_{z} z\right) \\
& H_{\phi}(\rho, \theta, z)=E_{o} \sqrt{\frac{\epsilon_{o}}{\mu_{o}}} \frac{k}{k_{r}} J_{1}\left(k_{01} \rho / R\right) \sin \left(k_{z} z\right)
\end{aligned}
$$

with $k_{r}=k_{01} / R, k_{01}=2.4048 \ldots$ and $k_{z}=\pi / L_{z}$. The volume of integration may be separated into two parts, the first estimating the frequency shift due to the cylindrical portion of the paddle, the second, the shift due to the paddle itself. Assuming the boundaries follow constant coordinate surfaces for ease of integration, (reasonable only for small perturbations to the cavity geometry) both integrals are of the form:

$$
\Delta W=\int_{\mathcal{V}}\left(\mu_{o} H^{2}-\epsilon_{o} E^{2}\right) d V=\epsilon_{o} E_{o}^{2} \int_{-\alpha}^{\alpha} d \theta \int_{\rho_{1}}^{\rho_{2}} \rho\left(\kappa^{2} J_{1}^{2}\left(k_{01}\right)-J_{o}^{2}\left(k_{01}\right)\right) d \rho \int_{L_{z}-d}^{L_{z}+d} \sin \left(k_{z} z\right)^{2} d z
$$

where $\kappa \equiv \frac{k}{k_{r}}$. As the paddle is indeed a small perturbation on the exterior wall, the fields may be expanded in a Taylor series about $\rho=R$, to yield:

$$
\begin{aligned}
& J_{o}\left(k_{01} \rho / R\right) \sim-J_{1}\left(k_{01}\right) k_{01}(\rho / R-1)+\frac{J_{1}\left(k_{01}\right) k_{01}}{2}(\rho / R-1)^{2} \\
& J_{1}\left(k_{01} \rho / R\right) \sim J_{1}\left(k_{01}\right)+J_{1}\left(k_{01}\right)(\rho / R-1)-\frac{1}{2} J_{1}\left(k_{01}\right)\left(1-\frac{2}{k_{01}^{2}}\right) k_{01}^{2}(\rho / R-1)^{2}
\end{aligned}
$$


and the integrals evaluated (keeping terms to second order in $(\rho / R-1)$ ):

$$
\Delta W=\epsilon_{o} E_{o}^{2}[2 \alpha]\left[\left.J_{1}^{2}\left(k_{01}\right) R^{2} \frac{\kappa}{2}(\rho / R-1)(-1+3 \rho / R)\right|_{\rho_{1}} ^{\rho_{2}}\right]\left[d+\frac{L_{z}}{2 \pi^{\frac{3}{2}}} \sin \left(\frac{d \pi^{\frac{3}{2}}}{L_{z}}\right)\right]
$$

where the notation $\Delta W$ is understood to mean the difference of the displaced magnetic and electric field energies $\Delta W_{H}-\Delta W_{E}$. The cylindrical portion of the paddle may be approximated by a square cross section plunger of equal cross sectional area, yielding the contribution:

$$
\Delta W^{c}=\epsilon_{o} E_{o}^{2}\left[\frac{a \sqrt{\pi}}{R}\right]\left[\kappa R J_{1}^{2}\left(k_{01}\right)\left(\delta-\frac{3 \delta^{2}}{2 R}\right)\right]\left[a+\frac{L_{z}}{2 \pi^{\frac{3}{2}}} \sin \left(\frac{a \pi^{\frac{3}{2}}}{L_{z}}\right)\right]
$$

which is, of course, independent of the paddle's orientation with respect to the electric field polarization. The contribution due to the paddle when aljgned with the field is:

$$
\Delta W_{\mathrm{l}}^{p}=\epsilon_{o} E_{o}^{2}\left[\frac{c}{R}\right]\left[\kappa J_{1}^{2}\left(k_{01}\right)\left(R b-\frac{3 b^{2}}{2}-3 b \delta\right)\right]\left[a+\frac{L_{z}}{2 \pi^{\frac{3}{2}}} \sin \left(\frac{a \pi^{\frac{3}{2}}}{L_{z}}\right)\right]
$$

and when perpendicular to the field is:

$$
\Delta W_{\perp}^{p}=\epsilon_{o} E_{o}^{2}\left[\frac{2 a}{R}\right]\left[\kappa J_{1}^{2}\left(k_{01}\right)\left(R b-\frac{3 b^{2}}{2}-3 b \delta\right)\right]\left[\frac{c}{2}+\frac{L_{z}}{2 \pi^{\frac{3}{2}}} \sin \left(\frac{\frac{c}{2} \pi^{\frac{3}{2}}}{L_{z}}\right)\right]
$$

where $a, b, c$, and $\delta$ are defined in figure 11 above. With the total stored energy in a $T M_{010}$ pill box field expressed as:

$$
U=\frac{\epsilon_{o}}{4} \pi R^{2} L_{z}\left(J_{1}^{2}\left(k_{01}\right)+\left(\frac{k_{z}}{k_{r}}\right)^{2} J_{2}^{2}\left(k_{01}\right)\right) E_{o}^{2}
$$

the frequency perturbation due to the entire paddle may be written:

$$
\begin{aligned}
& \delta \omega_{\|} \approx \omega_{\circ} \frac{1}{4 U}\left(\Delta W_{\|}^{p}+\Delta W^{c}\right) \\
& \delta \omega_{\perp} \approx \omega_{\circ} \frac{1}{4 U}\left(\Delta W_{\perp}^{p}+\Delta W^{c}\right)
\end{aligned}
$$

It is convenient to set the paddle dimensions to give a few megahertz frequency variation in $5 \mathrm{~mm}$ of insertion distance, and a frequency difference due to paddle rotation equal to three times the minimum settable frequency difference. By this choice, a large frequency adjustment range is available with coarse control via linear positioning and fine control via paddle rotation. Figure 12 shows the estimated tuning performance of the paddle over the useful range of motion. The main body of the paddle is chosen to have a radius $a=1.0$ $\mathrm{cm}$, which, when taken together with an assumed linear positioning precision of 20 microns yields a minimum frequency resolution of $28.0 \mathrm{kHz} / \mathrm{micron}$. Thus the paddle dimensions $b=1.0 \mathrm{~cm}$ and $c=3.0 \mathrm{~mm}$ are chosen to yield roughly $2 \times 28.0 \mathrm{kHz}$ over the 90 degree rotation of the paddle. Figure 12 reflects these dimensional choices. 


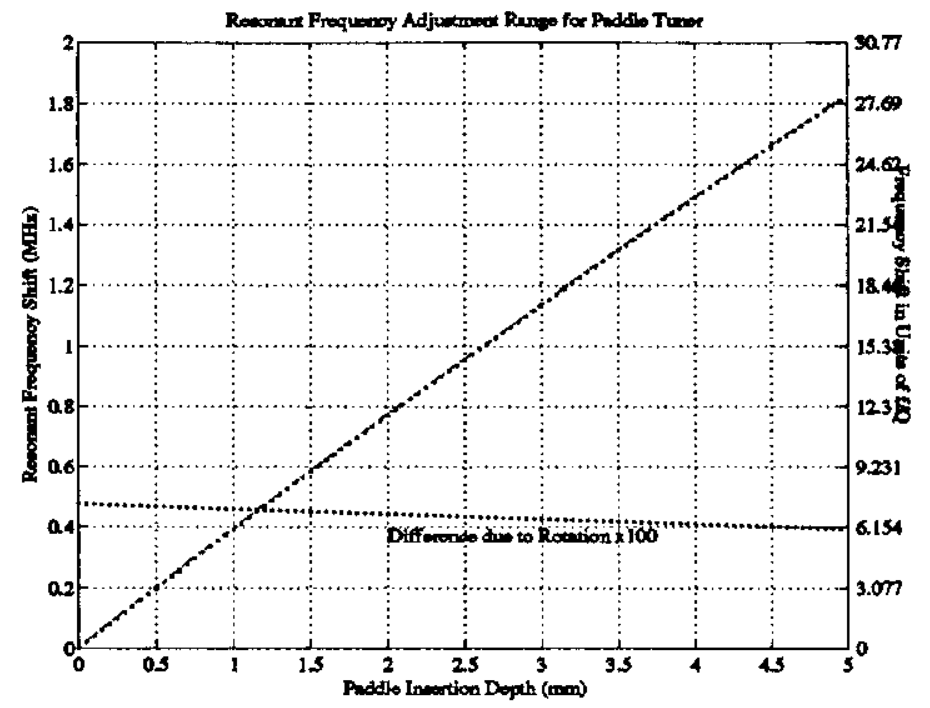

Figure 12: Frequency Tuning Performance of Paddle-Type Tuner

\subsection{Appendix B: Photocathode Excitation Laser Requirements}

Although a number of different methods of producing electrons have been developed and successfully used in electron guns, the highest current densities $\left(>1 \mathrm{kA} / \mathrm{cm}^{2}\right)$ possible with a laser driven photocathode are well matched to the high peak current requirements for the TTF and TESLA500 linacs. Additionally, some flexibility is available in the shaping of the charge distribution within the laser-generated beam, allowing some control over the nonlinear space charge contributions to the emittance.

A laser system suited to the requirements of the TTF and TESLA500 injectors is summarized in table 5 below. Laser pulse energies are listed for two photocathode materials which roughly represent the two extremes of photocathode performance: a metallic cathode (Yttrium is taken as an example), with low quantum efficiency (QE), and a semiconductor photocathode $\left(\mathrm{Cs}_{2} \mathrm{Te}\right)$ with excellent $\mathrm{QE}$. Restrictions on the timing jitter arise from the need to launch the bunch at a particular time during the $\mathrm{RF}$ cycle to minimize the $\mathrm{RF}$ contribution to the emittance growth, while restrictions on the amplitude jitter are needed to ensure consistent charge per bunch, and thus consistent beam loading in the downstream linac sections.

Discussions with members of industry have led to the conclusion that a laser matching the outlined requirements is technically feasible, although challenging. The conflicting requirements of high gain (for useful output pulse energy) and very long pulse trains (requiring little sag or fluctuation in the gain as subsequent pulses are amplified) will require state-of-the-art gain media, and potentially some form of closed-loop control system, as suggested by the researchers at the Max Born Institute.

Development of a laser satisfying the above parameters is underway at the Max Born Institut für Physik. A preliminary proposal for a three-stage Ti:Sapphire/LiSaF system is shown schematically in figure 13 on the next page. Pulses from a Ti:sapphire oscillator 


\begin{tabular}{|l|c|c|}
\hline \hline Parameter & \multicolumn{2}{|c|}{ Required Value } \\
\hline (Photocathode Material) & $C s_{2}$ Te & Metallic \\
\hline (Photocathode QE) & $1 \%$ & $0.05 \%$ \\
\hline Micropulse Energy & $>4 \mu \mathrm{J}$ & $>80 \mu \mathrm{J}$ \\
\hline Wavelength & \multicolumn{2}{|c|}{$<300 \mathrm{~nm}$} \\
\hline Pulse Length (FWHM) & \multicolumn{2}{|c|}{$8 \mathrm{ps}$} \\
\hline Micropulse Repetition Rate & \multicolumn{2}{|c|}{$1 \mathrm{MHz}$} \\
\hline Macropulse Length & $800-1000$ pulses \\
\hline Macropulse Repetition Rate & $10 \mathrm{~Hz}$ \\
\hline Timing Jitter & $<1 \mathrm{ps}$ \\
\hline Amplitude Jitter & $<5 \%$ \\
\hline Pointing Stability & $<100 \mu \mathrm{rad}$ \\
\hline \hline
\end{tabular}

Table 5: Laser requirements for the TTF/TESLA500 Photoinjectors

are selected to form the required pulse train structure, stretched, then amplified in two successive LiSaF gain stages and recompressed. For added amplitude control, a feed forward system allowing interpulse amplitude control surrounds the two doubling stages needed to produce the UV output. 


\section{Laser Concept for the TESLA Photocathode RF Gun}

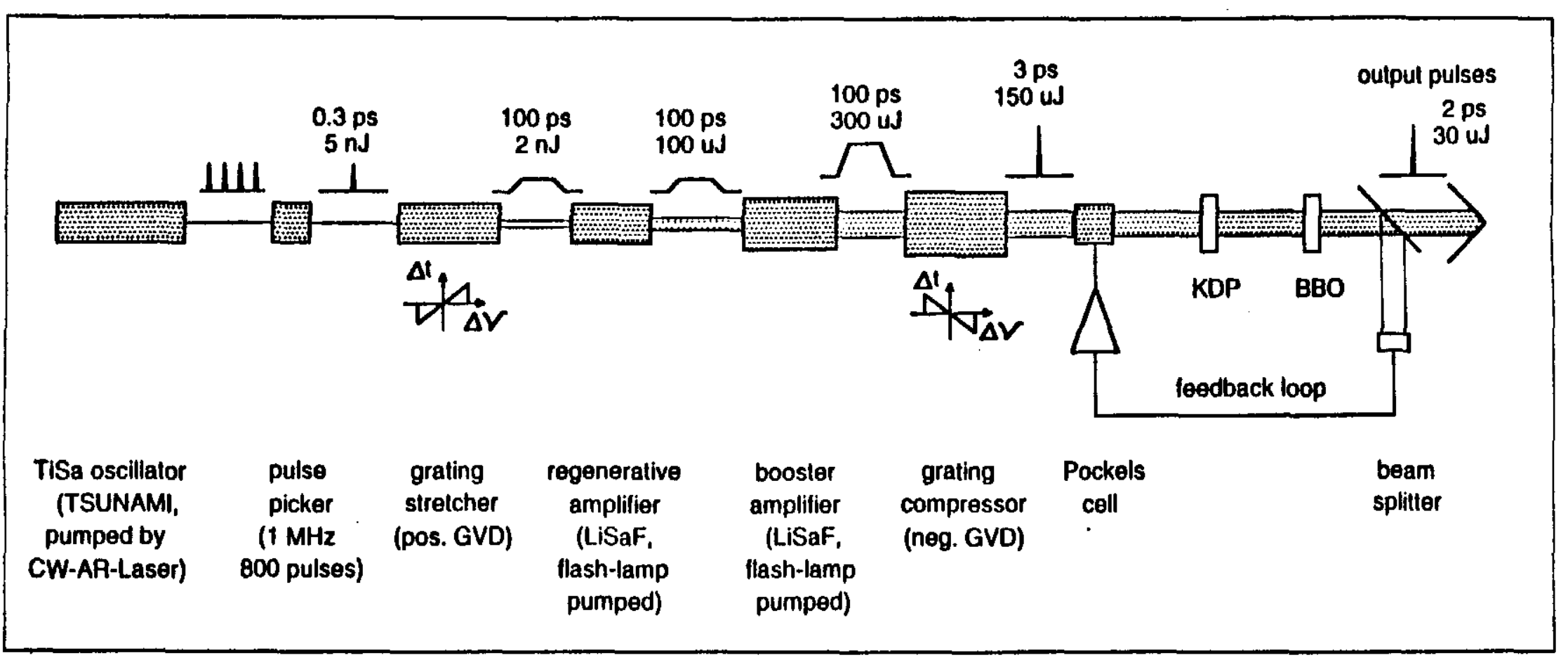




\subsection{Appendix C: Slit Based Emittance Measurement}

\section{Design Considerations for the UCLA PBPL Slit-based Phase Space Measurement Systems}

\section{J. Rosenzweig and G. Travish}

\section{February 22, 1994}

The phase space measurement system initially implemented by Spencer Hartman on the UCLA PBPL if photocathode gun has been upgraded, and a new system has been designed to measure the emittance at higher energy, after the emittance compensating drift and acceleration in the PWT linac. The purpose of this note is to describe the design criteria and physical principles involved in obtaining systems which provide the resolution in phase space measurements that we require. The final slit and detector hardware designs are included; the video data acquisition and analysis will remain nearly unchanged from Hartman's system.

The purpose of collimating the high intensity electron beam with the slits in these devices is two-fold. The first purpose is of course to separate the beam into many beamlets, whose intensity distribution at some downstream point can be measured to give the phase space distribution of the beam; the width of each beamlet gives a measure of the width of the transverse momentum distribution at each slit, and the centroid of the beamlets gives the correlated offset of the momentum distribution at each slit.

The second purpose is of prime importance for our beam, since it is space-charge dominated for almost all energies and beam-sizes of interest after the gun. This is quantified by comparing the space charge and emittance terms in the rms beam envelope equation in a drift space

$$
\sigma_{x}^{\prime \prime}=\frac{\varepsilon_{n}^{2}}{\gamma^{2} \sigma_{x}^{3}}+\frac{4 I}{\gamma^{3} I_{0}\left(\sigma_{x}+\sigma_{y}\right)}
$$

where $I$ is the peak beam current, $I_{0}=e c / r_{c}$ is the Alfven current, the beam is assumed relativistic ( $\gamma \gg 1, \beta=1), \varepsilon_{n}$ is the normalized emittance, and, of course, an analogous equation exists for $\sigma_{y}$. Now, taking the ration of the second to the first terms on the right hand side of the envelope equation, and assuming a round beam $\left(\sigma_{x}=\sigma_{y} \equiv \sigma_{0}\right)$, we have a measure of the degree of space charge dominance over emittance in driving the evolution of the beam envelope, 


$$
R_{0}=\frac{2 I \sigma_{0}^{2}}{I_{0} \gamma \varepsilon_{n}^{2}}
$$

One can see that for the relevant energies in our beamlines and experiments, that our high brightness beams are space-charge dominated $\left(R_{0} \gg 1\right)$ except near small waists, and thus linear transport theory cannot be used to measure the emittance (e.g. quad scanning). Collimation with slits mitigates this situation, however, by creating low current, small $\sigma_{x}$ beamlets which have the same uncorrelated "temperature" as the original beam. Noting that the rms size of a uniform beamlet created by a vertical slit of width $d$ is $\sigma_{x}=d / \sqrt{12}$, and assuming $\sigma_{x} \gg \sigma_{y}$, we have a space-charge dominance ratio for the beamlets,

$$
R_{b}=\sqrt{\frac{2}{3 \pi}} \frac{I}{Y_{0}}\left(\frac{d}{\varepsilon_{n}}\right)^{2} .
$$

This is ratio is similar in slit size scaling to that found by Hartman for round holes. For our case, at low energy ( $E=4 \mathrm{MeV}, I=200 \mathrm{~A}, \varepsilon_{n}=4 \mathrm{~mm}$-mrad), it is acceptable to have $d=50 \mu \mathrm{m}$ slits (see the calculation sheet included as an Appendix), that is $R_{b} \ll<1$, and the beamlets are emittance dominated. It is apparent that, at least from this point of view, that at high energy the same slit width will be adequate, as the ratio scales as $\gamma^{-1}$.

There are, in fact, other design considerations which impact on the choice of slit width, having to do with the angular acceptance of the slits. The depth of the material (stainless steel) used to intercept the beam is dictated by our desire to either stop the beam or scatter it sufficiently so that it doesn't affect the measurement of the nonintercepted beamlets. The stopping distance of the beam is approximately

$$
L_{s}=E / \frac{d E}{d t} \approx \frac{E(\mathrm{MeV})}{1.5\left(\mathrm{MeV}-\mathrm{cm}^{2} \mathrm{~g}^{-1}\right) \rho\left(\mathrm{g}-\mathrm{cm}^{-3}\right)}
$$

for an initially minimum ionizing particle. It is straightforward to stop a $4 \mathrm{MeV}$ beam, but with a $16 \mathrm{MeV}$ beam, the length of the slits becomes impractical. The beam scatters off of nuclei as it slows down from ionization losses, and the final rms angle associated with the beam after propagating a distance $L$ in the stopping material is approximately 


$$
\theta_{\alpha c}=\frac{21}{E(\mathrm{MeV})} \sqrt{\frac{L_{s}}{L_{r}}\left(\frac{1}{L_{s}-L}-1\right)},
$$

where $L_{r}$ is the radiation length in the material $(1.4 \mathrm{~cm}$ in steel). If we require a multiple scatter angle of approximately unity, then a $16 \mathrm{MeV}$ beam will need about $5 \mathrm{~mm}$ of steel to intercept and scatter it; this is in fact the design value we have chosen.

Once this length has been chosen, one can examine the angular acceptance of the slits. The first thing one needs to do is to specify an rms beam angle associated with the finite beam emittance, which assuming we place the slits at a waist, is

$$
\phi=\frac{\varepsilon_{n}}{\gamma \sigma_{0}} .
$$

This angle, which for a beam size of $\sigma_{0}=1.5 \mathrm{~mm}$, with $E=4 \mathrm{MeV}, \varepsilon_{n}=4 \mathrm{~mm}$-mrad, is $\phi=0.5 \mathrm{mrad}$. This should be much smaller than the half angle of the slit aperture, as it is for our case, in which $d / 2 L=5 \mathrm{mrad}$.

The slit separation $w$ is chosen to be much larger than the slit width $d$ and smaller than the beam size, to ensure that we can resolve the beam. In our case, the slit width is taken to be $0.75 \mathrm{~mm}$. This width must also be consistent with not allowing the beamlets to overlap at the detecting phosphor, a condition which depends on the distance of the drift to the phosphor $L_{d}$. The ratio to the beamlet widths to their separation, which should be much smaller than unity, is

$$
R_{w s}=2 \frac{L_{d} \phi}{w}
$$

while the ratio of the beamlet rms size at the phosphor to its size at the slit, which should be larger than one to achieve resolution of the uncorrelated angular spread in the beam, is

$$
R_{s p}=\sqrt{12} \frac{L_{d} \phi}{d} .
$$

Since one of these ratios should be small and the other large compared to unity, if we set their geometric average equal to unity $\left(R_{a p} R_{w s}=1\right)$, we can optimize the drift length to be 


$$
L_{d}=\frac{\sqrt{d w}}{3^{1 / 4} 2 \phi}
$$

which for our case yields $L_{d}=85 \mathrm{~cm}$. This optimum is of course quite broad, so we are free to choose a more convenient value as long as it is with a factor of two or so; we have chosen $L_{d}=60 \mathrm{~cm}$ for the $16 \mathrm{MeV}$ system.

Once the drift length is specified, there is another criterion which should be examined for the diagnostic to give unambiguous results, that the contribution to the measured emittance from the residual space-charge forces between beamlets is smaller than that due to the true uncorrelated angular distribution at the slits. Again assuming the slits are at a waist (this gives the highest estimate of the space-charge effect), we have

$$
R_{b}^{\prime}=\frac{2 I}{\gamma^{2} I_{0}} \frac{d L_{d}}{w \varepsilon_{n}}
$$

Again, this quantity must be much smaller than one. For our present design it is about 0.25 , but it should be noted for Hartman's measurements it was in fact greater than one.

The subject of slit scattering is a bit complicated, but a detailed calculation using EGS is not necessary if estimates that the signal to noise due to slit scatter is not of order 100 or less. Theoretical guidelines in this calculation have been developed by Courant (E.D. Courant, Rev. Sci. Instr. 22, 1003 (1951)) and Burge and Smith (E.J. Burge and D.A. Smith, Rev. Sci. Instr. 331371 (1962)). Modifying Courant's criterion for energy discrimination, we pick the effective depth of the maximum of the relevant slit scattered flux to occur when the multiple scattering angle is equal to the acceptance half-angle of the slits,

$$
l_{\text {cff }}=L_{r}\left(\frac{21 \cdot d}{E(\mathrm{MeV}) \cdot 2 l}\right)^{2}
$$

and the increase in effective slit width is given by

$$
d_{e f f}=\frac{2}{\sqrt{3 \pi}} \frac{l_{e f}^{3 / 2}}{w_{c}},
$$

where 


$$
w_{c}^{2}=\frac{A}{Z^{2} \pi N_{A} \rho}\left(\frac{E}{2 e^{2}}\right)^{2} \ln \left(181 \cdot Z^{1 / 3}\right)^{-1}
$$

and $N_{A}$ is Avogadro's number. The minimum signal to noise for the detected beam intensity at the phosphor is therefore

$$
\frac{S}{N} \geq \frac{\sqrt{3 \pi} d w_{c}}{2 d_{d \delta} l_{d f}^{3 / 2}}
$$

For our case, this is greater than $10^{4}$. It should be noted however, that a misalignment of the slits can generate anomalously large slit scattering effects, and thus care must be taken to avoid this situation.

The layout of the emittance slits in the beamline, and their hardware design are shown in an Appendix. More care has been taken in the machining specifications for the slits, to ensure that they are flat over the entire surface parallel to the beam propagation. This is accomplished by electron discharge machining (EDM), and is essential for our slit systems (and others being developed for Argonne and Fermilab which have even narrower slit requirements). The slits are now mounted on a rotatable actuator which is driven by a stepping motor, eliminating the tedious job of aligning the slits by hand. 


\subsection{Appendix D: Electrical Dimension Specifications and Tolerances}

Electrical dimensions and tolerances derived from physical constraints on the resonant frequency, coupling constant, and normalized structure impedance are listed in table 6 below. Engineering drawings of the cold test model components follow on the subsequent pages.

Constraints on the cavity resonant frequency, $f_{o}$, set by the available tuning range of the paddle tuners, cathode plunger, and thermal control system, are necessary to have the resonant frequency of the structure fall at the desired frequency, and to avoid field imbalance (viz. the ratio $E_{z, \max }^{\text {half }} / E_{z, \max }^{f \text { ull }}$ ) problems due to a mismatch in the individual cavity resonant frequencies. As the outer walls of the cavity experience substantial magnetic field, the frequency of the cavity varies rapidly with radius, placing tight tolerances on the radii. Tolerances listed below derive from the frequency constraint $\Delta f \leq 1 \mathrm{MHz}$.

The coupling constant $\gamma$ prescribes the frequency separation of the zero and pi modes of the structure, and controls to what extent movement of the photocathode perturbs the field balance. So long as gamma is large enough to provide for well separated zero and pi mode frequencies, (ie. the separation is greater than a half $Q$-width: $\omega_{\pi}-\omega_{0} \geq \frac{\omega_{0}}{2 Q}$, where $\omega_{0}$ is the resonant frequency of the uncoupled cavity) small changes in $\gamma$ will not appreciably alter the field balance. Correspondingly, the tolerance on the coupling constant is generous at $\Delta \gamma \leq .05 \%$.

The normalized structure impedance, $\beta$, determines the behavior of the photoinjector when coupled to an RF source. Optimal power transfer from RF source to accelerating structure occurs when $\beta \equiv \frac{P_{\text {cavities }}+P_{\text {beam }}}{P_{\text {transmission system }}}=1$. Tolerance on the physical dimensions of the coupling hole are tight, owing to the rapid $\left.(\alpha \text { (slot area })^{6}\right)$ variation of $\beta$. Stipulating that the reflected power from the cavity not exceed $1 \%$ requires $0.818<\beta<1.222$.

\begin{tabular}{|l|c|c|c|}
\hline Dimension & Nominal Value & Tolerance & Quantity Principally Affected \\
\hline radius of half cell & 8.904 & $\pm 0.0068=2.7 \mathrm{mil}$ & $f_{0}^{H C}$ \\
\hline radius of full cell & 8.884 & $\pm 0.0068=2.7 \mathrm{mil}$ & $f_{0}^{F C}$ \\
\hline radius of iris & 2.0 & $\pm 0.0833=33 . \mathrm{mil}$ & $\gamma$ \\
\hline thickness of iris & 1.5 & $\pm 0.0142=5.6 \mathrm{mil}$ & $\gamma$ \\
\hline length of coupling slot & 6.0 & $\pm 0.0500=19 . \mathrm{mil}$ & $\beta$ \\
\hline width of coupling slot & 2.0 & $\pm 0.0167=6.6 \mathrm{mil}$ & $\beta$ \\
\hline depth of coupling slot & 0.5 & $\pm 0.0548=22 . \mathrm{mil}$ & $\beta$ \\
\hline
\end{tabular}

All dimensions in centimeters unless specified

Table 6: Key Electrical Dimension Specifications for Photoinjector 
Side View Center Cut
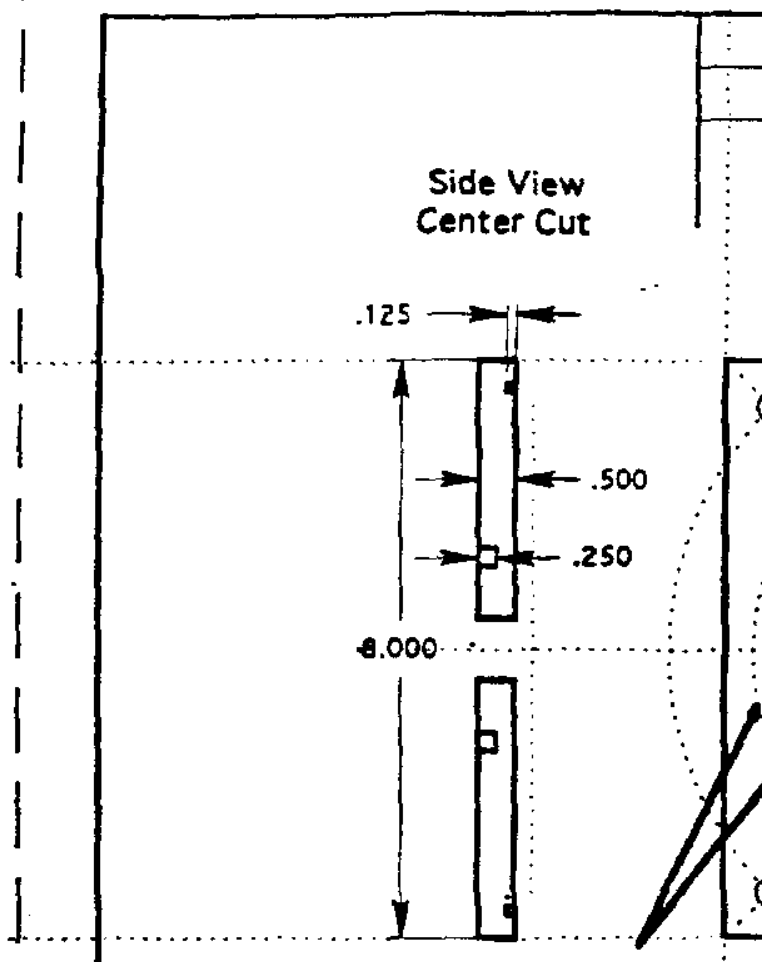

Arnular region bermeen arrows (onty right thet surface in side viow above) must have 15

surface polsh or better

All tolerances $+1-0.005$ excape where indicated

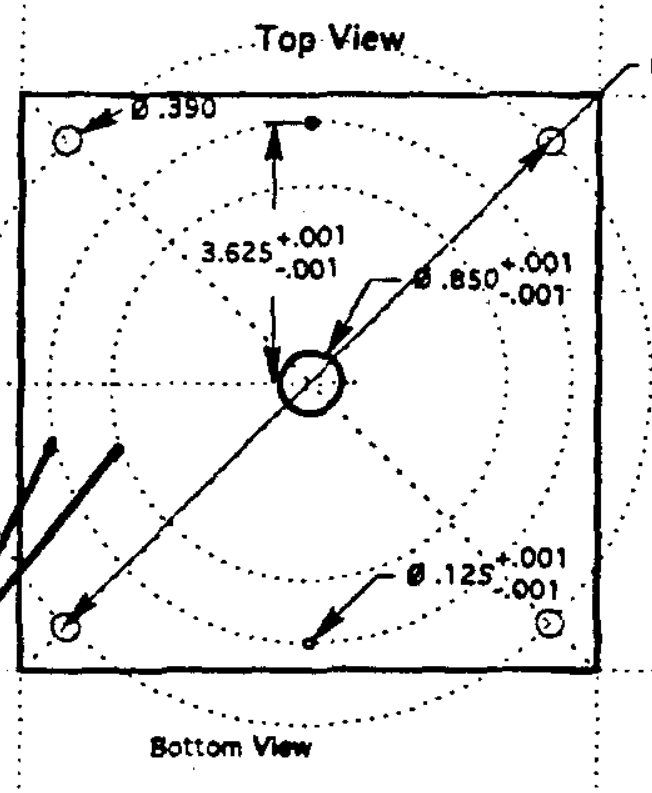

9.500

$0.375^{\circ}$ thrs

holes (4), on

9.5" circle

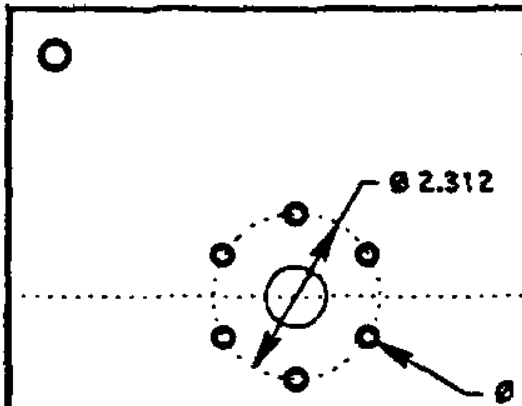

1/4-28 tapoed holes

(6) on 2-3/8 bott cirete

.250

0

o

o

.000

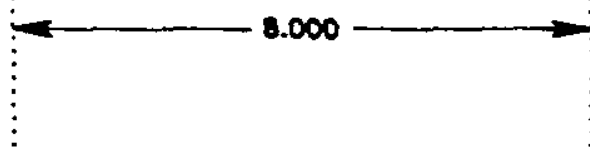

\begin{tabular}{|c|c|c|}
\hline Drewn By. J. Rosonzwipg & & Ashlar \\
\hline Suly 13,1994 & \multirow{2}{*}{\multicolumn{2}{|c|}{ RF gun cathode plane }} \\
\hline Qty noeded: 1 & & \\
\hline ITF Gun Cold Test Mosid & A $\operatorname{san}^{2}$ sat 1:3.5 & Materiat: Brass \\
\hline
\end{tabular}




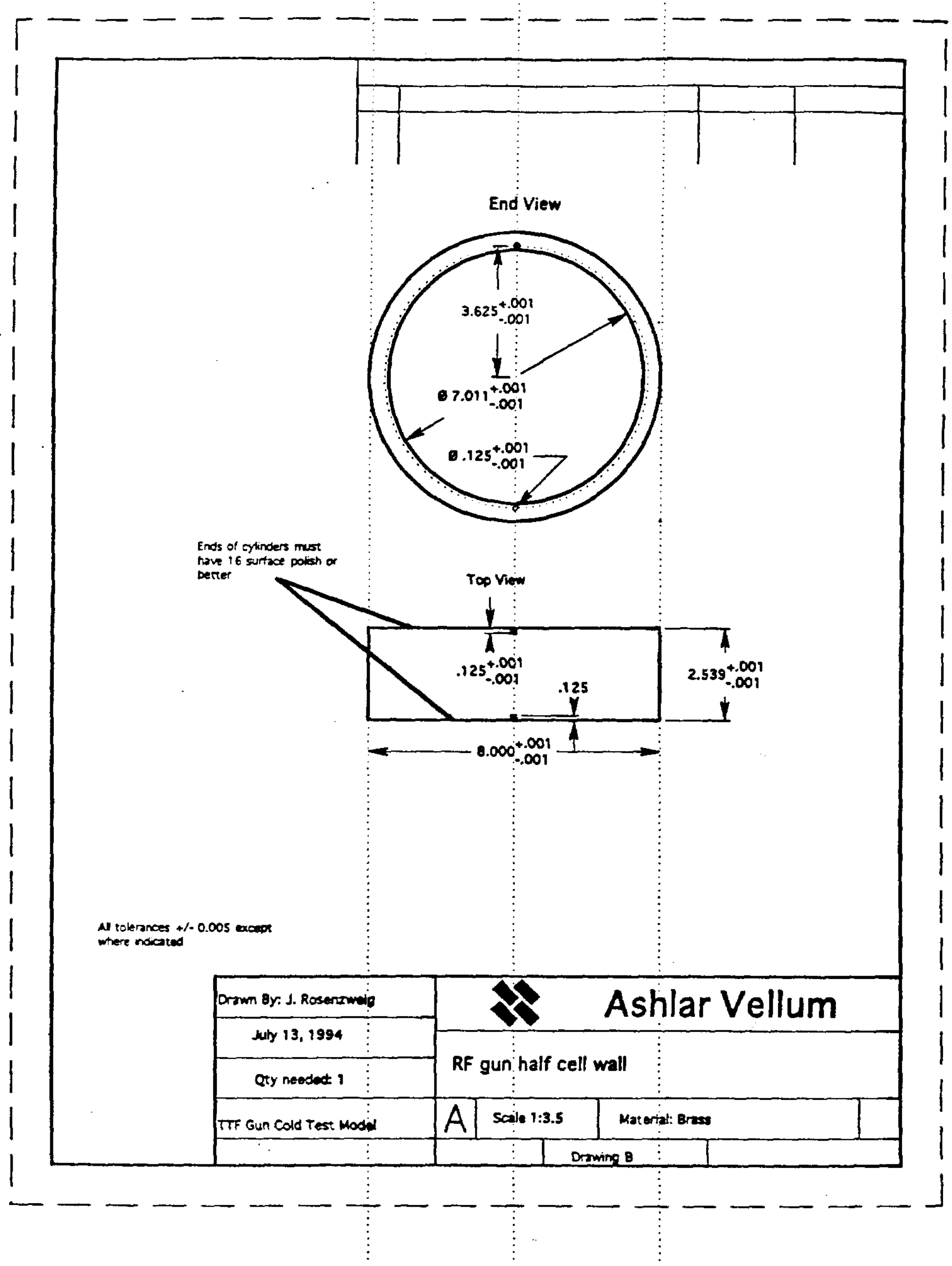




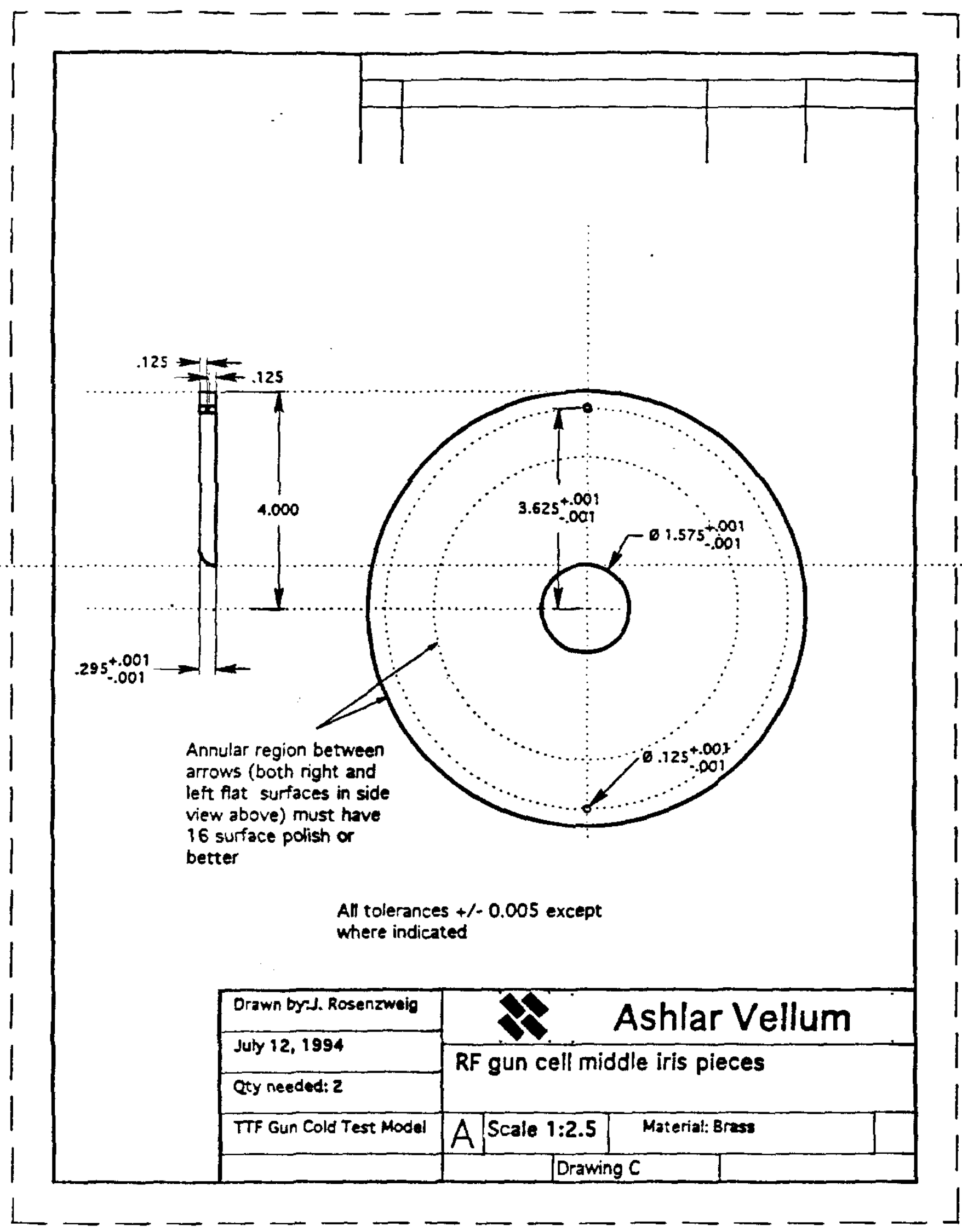




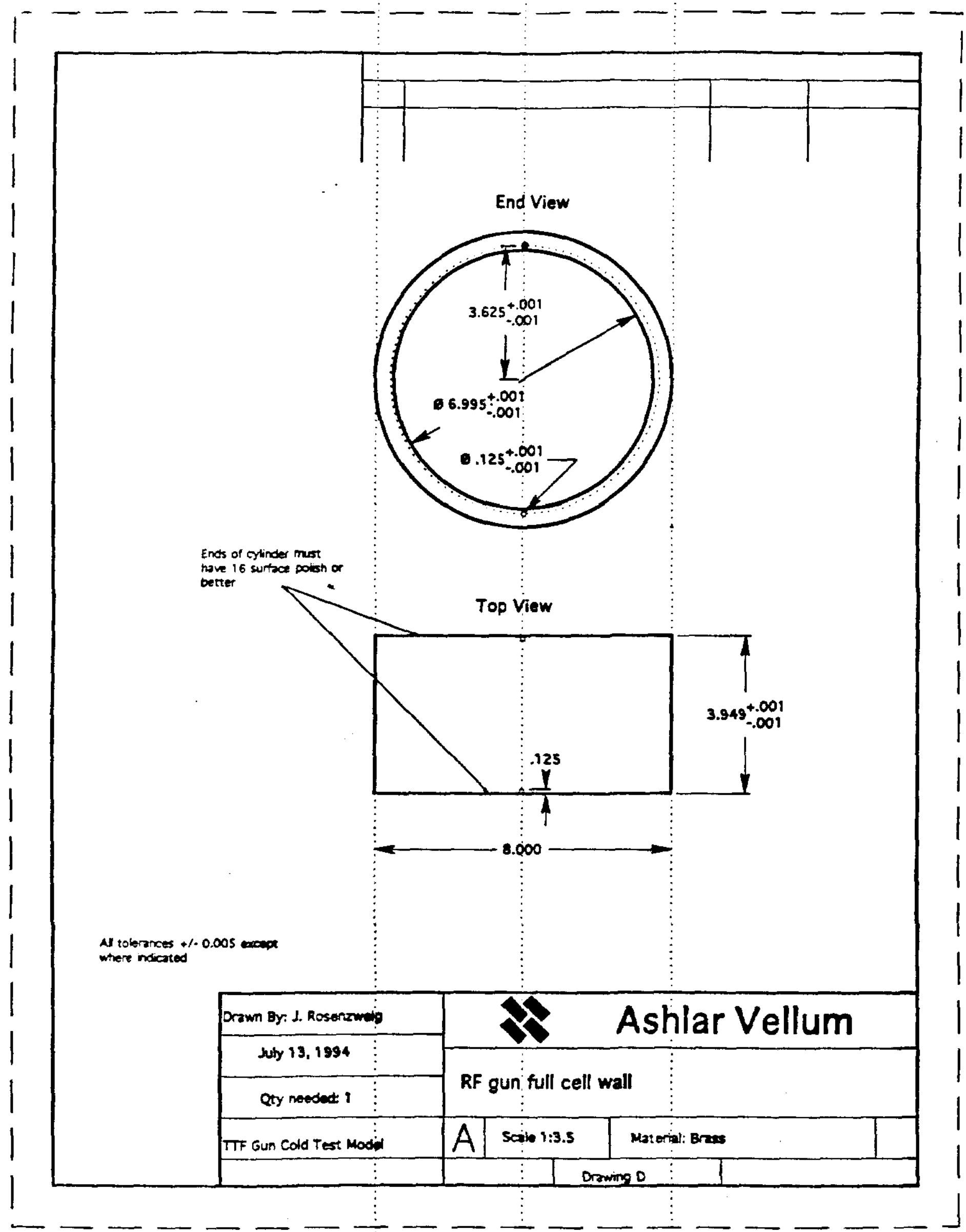




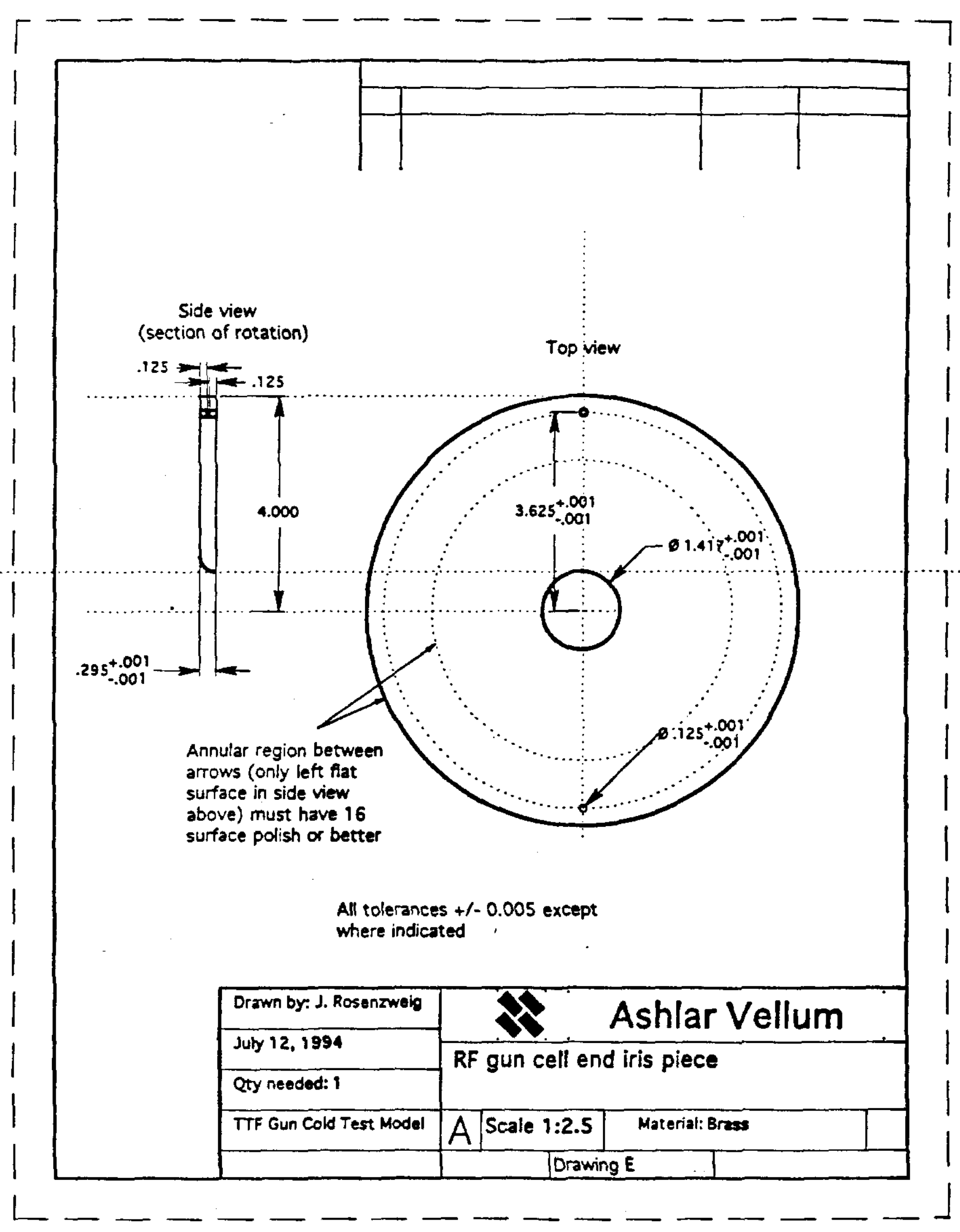


Side View

Center Cut
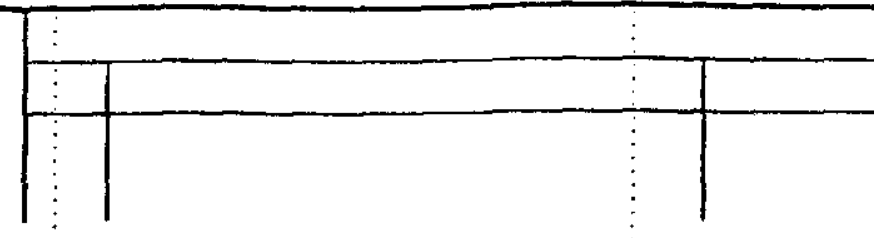

9.500

$0.375^{\prime \prime}$ thru holes (4), on

9.5" circle

Al tolerances $+1 \cdot 0.005$ except where indicated

Amular region bermoen arrous lanly right then sutace in side view

abovel mast have 16

sufface polish or better

. Top View.

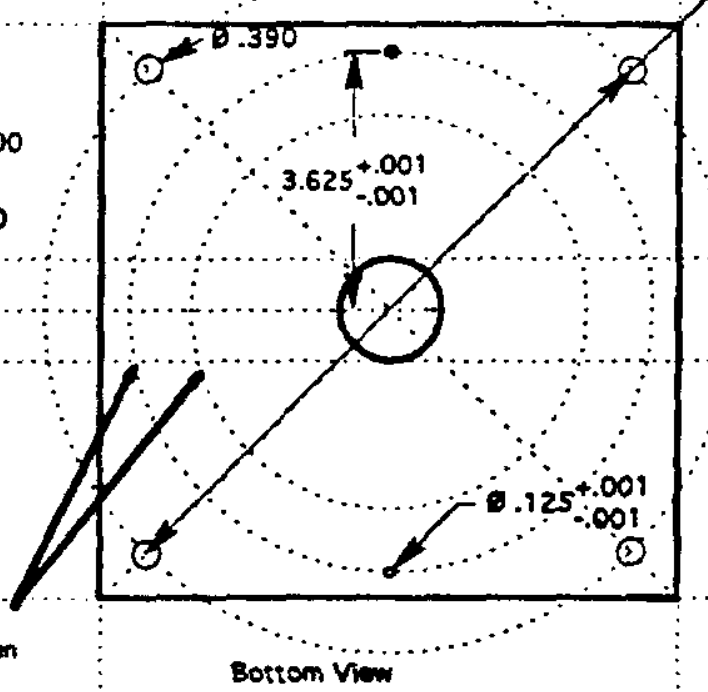

\section{.}
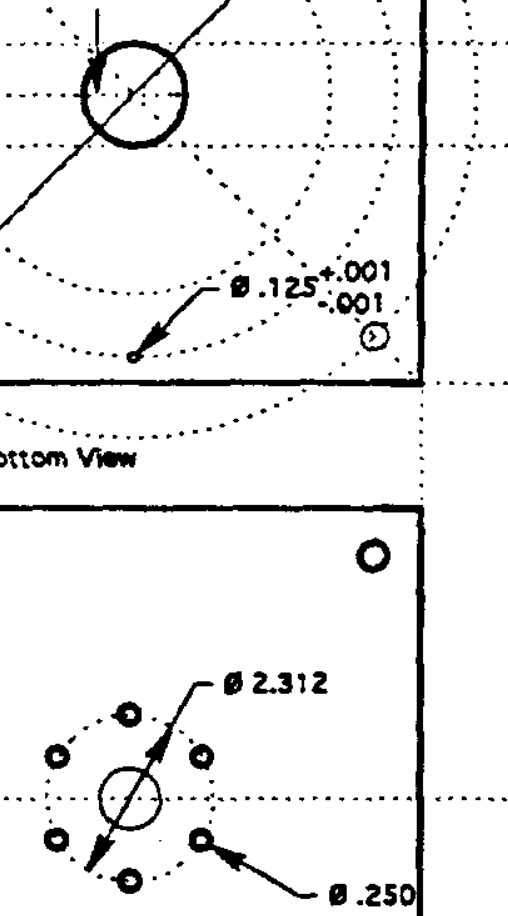

1/4-28 tapped holes

(6) on 2-3/8 bolt crove

.250

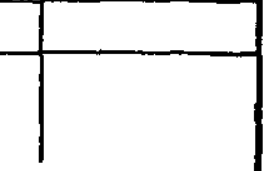

